JOURNAL OF

FUNCTION SPACES AND APPLICATIONS

Volume 4, Number 2 (2006), 113-144 (c) 2006, Scientific Horizon http://www.jfsa.net

\title{
Characterization of Riesz and Bessel potentials on variable Lebesgue spaces
}

\author{
Alexandre Almeida and Stefan Samko \\ (Communicated by Vakhtang Kokilashvili)
}

2000 Mathematics Subject Classification. 46E35, 26A33, 42B20, 47B38.

Keywords and phrases. Lebesgue space with variable exponent, hypersingular integral, Riesz potential, Bessel potential.

\footnotetext{
Abstract. Riesz and Bessel potential spaces are studied within the framework of the Lebesgue spaces with variable exponent. It is shown that the spaces of these potentials can be characterized in terms of convergence of hypersingular integrals, if one assumes that the exponent satisfies natural regularity conditions. As a consequence of this characterization, we describe a relation between the spaces of Riesz or Bessel potentials and the variable Sobolev spaces.
}

\section{Introduction}

The Lebesgue spaces $L_{p(\cdot)}$ with variable exponent and the corresponding Sobolev spaces $W_{p(\cdot)}^{m}$ have been intensively investigated during the last years. We refer to the papers [16], [27], where the basics of such spaces were developed, to the papers [9], [23], where the denseness of nice functions

The first author is partially supported by Unidade de Investigação "Matemática e Aplicações" of Universidade de Aveiro, through Programa Operacional "Ciência, Tecnologia e Inovação" (POCTI) of the Fundação para a Ciência e a Tecnologia (FCT), cofinanced by the European Community Fund FEDER. 
in Sobolev variable spaces was considered, and to the papers [3], [5], [7], [8], [13], [15],[18], [21], [22] and the recent preprints [2], [4] and references therein, where various results on maximal, potential and singular operators in variable Lebesgue spaces were obtained (see also the surveys [12], [25]). The interest to the Lebesgue spaces with variable exponent during the last decade was in particular roused by applications in problems of fluid dynamics, elasticity theory and differential equations with non-standard growth conditions (see [8], [19]).

We deal with the spaces of Riesz and Bessel potentials with densities in the spaces $L_{p(\cdot)}\left(\mathbb{R}^{n}\right)$. For the constant $p$, it is known that the left inverse operator to the Riesz potential operator $\mathcal{I}^{\alpha}$ within the frameworks of the spaces $L_{p}\left(\mathbb{R}^{n}\right)$ may be constructed in terms of the hypersingular integrals, and the range $\mathcal{I}^{\alpha}\left[L_{p}\right]$ is described in terms of convergence of those hypersingular integrals, see [20], [24]. The extension of the statement on the inversion to the case of variable exponents was recently given in [1].

In this paper we give a description of the range of the Riesz and Bessel potential operators, $\mathcal{I}^{\alpha}\left[L_{p(\cdot)}\right]$ and $\mathcal{B}^{\alpha}\left[L_{p(\cdot)}\right]$, respectively, in terms of convergence of hypersingular integrals. As a consequence, we also establish a connection of the spaces of Riesz and Bessel potentials with the Sobolev spaces $W_{p(\cdot)}^{m}$. This partially extends the known results for constant $p$ (see [24]) to the variable exponent setting.

The paper is organized as follows. In Section 2 we provide notation and necessary preliminaries and auxiliary results which will be often used throughout the text. The first main result, Theorem 3.2, given in Section 3 contains a characterization of the space of Riesz potentials in terms of fractional derivatives. In Section 4 we consider some spaces of fractional smoothness defined in terms of hypersingular integrals and study their connection with the space of Riesz potentials, the main results being given in Theorems 4.1 and 4.4. The study of the space of Bessel potentials on $L_{p(\cdot)}$ and its description are made in Section 5, see Theorem 5.7. In the last section the connection between the spaces of potentials and the Sobolev spaces with variable exponent is studied.

Throughout the paper, we shall consider standard notation or it will be properly introduced whenever needed.

\section{Preliminaries}

As usual, $C_{0}^{\infty}\left(\mathbb{R}^{n}\right)$ stands for the class of all $C^{\infty}$ functions on $\mathbb{R}^{n}$ with compact support. By $\mathcal{S}\left(\mathbb{R}^{n}\right)$ we denote the Schwartz class of all rapidly decreasing $C^{\infty}$-functions on $\mathbb{R}^{n}$, and by $\mathcal{S}^{\prime}\left(\mathbb{R}^{n}\right)$ its dual. For $\varphi \in \mathcal{S}\left(\mathbb{R}^{n}\right)$, 
by $F \varphi($ or $\widehat{\varphi})$ we denote the Fourier transform of $\varphi$,

$$
(F \varphi)(\xi)=\int_{\mathbb{R}^{n}} e^{i x \cdot \xi} \varphi(x) d x, \quad \xi \in \mathbb{R}^{n} .
$$

By $\mathcal{W}_{0}\left(\mathbb{R}^{n}\right)$ we denote the class of Fourier transforms of integrable functions.

By $\Phi^{\prime}\left(\mathbb{R}^{n}\right)$ we denote the topological dual of the Lizorkin space $\Phi\left(\mathbb{R}^{n}\right)$ consisting of all functions $\varphi \in \mathcal{S}\left(\mathbb{R}^{n}\right)$ such that $\left(D^{\beta} \widehat{\varphi}\right)(0)=0$, for all $\beta \in \mathbb{N}_{0}^{n}$, where $D^{\beta}$ is the usual partial derivative. Two elements of $\mathcal{S}^{\prime}\left(\mathbb{R}^{n}\right)$ differing by a polynomial are indistinguishable as elements of $\Phi^{\prime}\left(\mathbb{R}^{n}\right)$ (see [24], Section 2.2).

By $C$ (or $c$ ) we denote a general positive constant whose value is irrelevant and may change at different occurrences.

\subsection{On Lebesgue spaces with variable exponent}

A detailed discussion of properties of the variable Lebesgue spaces may be found in the papers [9], [10], [16], [27]. We recall here some important tools and definitions which will be used throughout this paper.

Let $p: \mathbb{R}^{n} \rightarrow[1, \infty)$ be a (Lebesgue) measurable function. Put

$$
\bar{p}:=\operatorname{ess} \sup _{x \in \mathbb{R}^{n}} p(x) \quad \text { and } \quad \underline{p}:=\operatorname{ess} \inf _{x \in \mathbb{R}^{n}} p(x) .
$$

By $L_{p(\cdot)}\left(\mathbb{R}^{n}\right)$ we denote the space of all measurable functions $f$ on $\mathbb{R}^{n}$ such that the modular

$$
I_{p(\cdot)}(f):=\int_{\mathbb{R}^{n}}|f(x)|^{p(x)} d x
$$

is finite. Under this definition, this is a linear space if and only if $\bar{p}<\infty$, and we only consider bounded exponents. $L^{p(\cdot)}\left(\mathbb{R}^{n}\right)$ is a Banach space endowed with the norm

$$
\|f\|_{p(\cdot)}:=\inf \left\{\lambda>0: I_{p(\cdot)}\left(\frac{f}{\lambda}\right) \leq 1\right\}, \quad f \in L_{p(\cdot)}\left(\mathbb{R}^{n}\right) .
$$

This space inherits some properties from the classical Lebesgue spaces with constant exponent. In fact, under the additional assumption $\underline{p}>1$, $L_{p(\cdot)}\left(\mathbb{R}^{n}\right)$ is uniformly convex, reflexive and its dual space is (isomorphic to) $L_{p^{\prime}(\cdot)}\left(\mathbb{R}^{n}\right)$, where $p^{\prime}(\cdot)$ is the natural conjugate exponent given by $\frac{1}{p(x)}+\frac{1}{p^{\prime}(x)} \equiv 1$. An important property of this space is that the convergence in norm is equivalent to the modular convergence: given $\left\{f_{k}\right\}_{k \in \mathbb{N}_{0}} \subset$ $L_{p(\cdot)}\left(\mathbb{R}^{n}\right)$, then $\left\|f_{k}\right\|_{p(\cdot)} \rightarrow 0$ if and only if $I_{p(\cdot)}\left(f_{k}\right) \rightarrow 0$, as $k \rightarrow \infty$. Nevertheless, this generalized space has some undesirable properties. As 
mentioned before, $L_{p(\cdot)}\left(\mathbb{R}^{n}\right)$ is not translation invariant contrarily to its classical counterpart.

Similarly to the classical case, one defines the Sobolev space of variable exponent $W_{p(\cdot)}^{m}\left(\mathbb{R}^{n}\right), m \in \mathbb{N}_{0}$, as the space of all measurable functions $f$ such that its (weak) derivatives $D^{\beta} f$ up to order $m$ are in $L_{p(\cdot)}\left(\mathbb{R}^{n}\right)$. This is a Banach space equipped with the norm

$$
\|f\|_{m, p(\cdot)}:=\sum_{|\beta| \leq m}\left\|D^{\beta} f\right\|_{p(\cdot)}, \quad f \in W_{p(\cdot)}^{m}\left(\mathbb{R}^{n}\right) .
$$

In order to emphasize that we are dealing with variable exponents, we always write $p(\cdot)$ instead of $p$ to denote an exponent function. In general, we will consider function spaces defined on the whole Euclidean space $\mathbb{R}^{n}$. So, in what follows, we shall omit the " $\mathbb{R}^{n}$ " from their notation.

\subsection{The maximal operator in $\mathrm{L}_{\mathbf{p}(\cdot)}$}

For a locally integrable function $g$ on $\mathbb{R}^{n}$, the Hardy-Littlewood maximal operator $\mathcal{M}$ is defined by

$$
\mathcal{M} g(x)=\sup _{r>0} \frac{1}{|B(x, r)|} \int_{B(x, r)}|g(y)| d y,
$$

where $B(x, r)$ denotes the open ball centered at $x \in \mathbb{R}^{n}$ and of radius $r>0$.

The boundedness of the maximal operator $\mathcal{M}$ was first proved by $\mathrm{L}$. Diening [5] over bounded domains, under the assumption that $p(\cdot)$ is locally $\log$-Hölder continuous, that is,

$$
|p(x)-p(y)| \leq \frac{C}{-\ln |x-y|}, \quad x, y \in \mathbb{R}^{n}, \quad|x-y| \leq 1 / 2 .
$$

He later extended the result to unbounded domains by supposing, in addition, that the exponent $p(\cdot)$ is constant outside some large fixed ball. The general case of the exponent $p(\cdot)$ non-constant at infinity, was considered in [18], where some integral condition was imposed and in [3] where it was assumed that the exponent is log-Hölder continuous at infinity:

$$
|p(x)-p(y)| \leq \frac{C}{\ln (e+|x|)}, \quad x, y \in \mathbb{R}^{n}, \quad|y| \geq|x| .
$$

Condition (2.4) is equivalent to the logarithmic decay condition

$$
|p(x)-p(\infty)| \leq \frac{C}{\ln (e+|x|)}, \quad x \in \mathbb{R}^{n},
$$


where $p(\infty)=\lim _{|x| \rightarrow \infty} p(x)$.

Let $\mathcal{P}\left(\mathbb{R}^{n}\right)$ be the class of all exponents $p(\cdot), 1<\underline{p} \leq \bar{p}<\infty$, such that $\mathcal{M}$ is bounded on $L_{p(\cdot)}$. Recently, L. Diening [6] obtained a certain necessary and sufficient condition for the exponent $p(\cdot)$ to be in $\mathcal{P}\left(\mathbb{R}^{n}\right)$.

Although the space $L_{p(\cdot)}$ is not invariant with respect to translations, and Young's theorem in general fails in the case of variable exponents, in [23] it was shown that it is possible to use the mollifier technique within the framework of these spaces, if one assumes that the exponent $p(\cdot)$ satisfies the local logarithmic continuity condition (2.3).

The approximation problem via mollifiers is closely related to the boundedness of the maximal operator (see [29], III.2.2). In [5], Stein's theorem on convolutions was extended to the variable exponent setting as follows:

Theorem 2.1. Let $\varphi$ be an integrable function on $\mathbb{R}^{n}$ and define $\varphi_{\varepsilon}(\cdot)=\varepsilon^{-n} \varphi(\cdot / \varepsilon), \varepsilon>0$. Suppose that the least decreasing radial majorant of $\varphi$ is integrable, i.e., $A:=\int_{\mathbb{R}^{n}} \sup _{|y| \geq|x|}|\varphi(y)| d x<\infty$. Then

(i) $\sup _{\varepsilon>0}\left|\left(f * \varphi_{\varepsilon}\right)(x)\right| \leq 2 A(\mathcal{M} f)(x), f \in L_{p(\cdot)}, x \in \mathbb{R}^{n}$.

If $p(\cdot) \in \mathcal{P}\left(\mathbb{R}^{n}\right)$, then also

(ii) $\left\|f * \varphi_{\varepsilon}\right\|_{p(\cdot)} \leq c\|f\|_{p(\cdot)}$ (with $c$ independent of $\varepsilon$ and $f$ ) and, if in addition $\int_{\mathbb{R}^{n}} \varphi(x) d x=1$, then

(iii) $f * \varphi_{\varepsilon} \rightarrow f$ as $\varepsilon \rightarrow 0$ in $L_{p(\cdot)}$ and almost everywhere.

Theorem 2.1 is an important tool which allows us to obtain boundedness of various concrete convolution operators, even in the case where they are defined by the Fourier transform of their kernel.

\subsection{The Riesz potential operator on variable Lebesgue spaces}

We recall that the Riesz potential operator $\mathcal{I}^{\alpha}$ is defined by

$$
\mathcal{I}^{\alpha} g(x):=\int_{\mathbb{R}^{n}} \frac{g(y)}{|x-y|^{n-\alpha}} d y,
$$

where $g$ is a locally integrable function with an appropriate behavior at infinity and $0<\alpha<n$.

The $L_{p(\cdot)} \rightarrow L_{q(\cdot)}$-boundedness of $\mathcal{I}^{\alpha}$ on $L_{p(\cdot)}$ spaces was first considered in [22], where the Sobolev type theorem for bounded domains was proved under the assumption that the maximal operator is bounded in $L_{p(\cdot)}$. After L. Diening [5] has proved the boundedness of the maximal operator, the conditional Sobolev theorem in [22] became an unconditional statement. L. 
Diening [7] proved the Sobolev theorem on $\mathbb{R}^{n}$ for $p(\cdot)$ satisfying the local logarithmic condition (2.3) and constant at infinity.

Some weighted version of the Sobolev theorem for $\mathbb{R}^{n}$ with the power weight fixed to infinity, was obtained in [14]. Recently, C. Capone, D. CruzUribe and A. Fiorenza [2] proved the Sobolev theorem on arbitrary domains for $p(\cdot)$ non-constant at infinity. Their statement for the case of the whole space $\mathbb{R}^{n}$ runs as follows.

Theorem 2.2. Let $0<\alpha<n$ and let $1<p \leq \bar{p}<n / \alpha$. Assume also that $p(\cdot)$ satisfies the $\log$-Hölder conditions (2.3) and (2.4). Then there exists $c>0$ such that

$$
\left\|\mathcal{I}^{\alpha} f\right\|_{q(\cdot)} \leq c\|f\|_{p(\cdot)}, \quad f \in L_{p(\cdot)},
$$

where $q(\cdot)$ is the Sobolev exponent given by $\frac{1}{q(x)}=\frac{1}{p(x)}-\frac{\alpha}{n}, x \in \mathbb{R}^{n}$.

\subsection{Hypersingular integrals on $\mathrm{L}_{\mathrm{p}(\cdot)}$ spaces}

We refer to [24] and [26] for the theory of hypersingular integrals.

A typical hypersingular integral has the form

$$
\frac{1}{d_{n, \ell}(\alpha)} \int_{\mathbb{R}^{n}} \frac{\left(\Delta_{y}^{\ell} f\right)(x)}{|y|^{n+\alpha}} d y, \quad \alpha>0,
$$

where $\Delta_{y}^{\ell} f$ denotes the finite difference of order $\ell \in \mathbb{N}$ of the function $f$ and $d_{n, \ell}(\alpha)$ is a certain normalizing constant, which is chosen so that the construction in (2.7) does not depend on $\ell$ (see [24], Chapter 3, for details). It is well-known that the integral (2.7) exists (for each $x \in \mathbb{R}^{n}$ ) if $f \in \mathcal{S}\left(\mathbb{R}^{n}\right)$ and $\ell>\alpha$, for instance.

Following [24], we shall consider both a centered difference and a noncentered one in the construction of the hypersingular integral. However, when we write $\Delta_{h}^{m}$ without any specification we mean a non-centered difference. The important fact here is that the order $\ell$ should be chosen according to the following rule (as stated in [24], page 65), which will be always assumed in the sequel:

(1) in the case of a non-centered difference we take $\ell>2\left[\frac{\alpha}{2}\right]$ with the obligatory choice $\ell=\alpha$ for $\alpha$ odd;

(2) in the case of a centered difference we take $\ell$ even and $\ell>\alpha>0$.

In general, the integral in (2.7) may be divergent, and hence it needs to be properly defined. We interpret hypersingular operators as $\mathbb{D}^{\alpha}:=\lim _{\varepsilon \rightarrow 0} \mathbb{D}_{\varepsilon}^{\alpha}$, where $\mathbb{D}_{\varepsilon}^{\alpha}$ denotes the truncated hypersingular operator 


$$
\mathbb{D}_{\varepsilon}^{\alpha} f(x)=\frac{1}{d_{n, \ell}(\alpha)} \int_{|y|>\varepsilon} \frac{\left(\Delta_{y}^{\ell} f\right)(x)}{|y|^{n+\alpha}} d y, \quad \varepsilon>0 .
$$

Sometimes $\mathbb{D}^{\alpha}$ is also called the Riesz fractional derivative since it can be interpreted as a positive fractional power $(-\Delta)^{\frac{\alpha}{2}}$ of the minus Laplacian.

In what follows, the limit above is always taken in the sense of convergence in the $L_{p(\cdot)}$ norm. This makes sense in view of the Proposition 2.3 below.

Proposition 2.3. If $p(\cdot) \in \mathcal{P}\left(\mathbb{R}^{n}\right)$, then the truncated hypersingular integral operator $\mathbb{D}_{\varepsilon}^{\alpha}$ is bounded in $L_{p(\cdot)}$, for every $\varepsilon>0$.

Another important property of the truncated hypersingular integrals is the following uniform estimate:

Proposition 2.4. Let $0<\alpha<n$ and $p(\cdot) \in \mathcal{P}\left(\mathbb{R}^{n}\right)$ with $\bar{p}<\frac{n}{\alpha}$. Then the compositions $\mathbb{D}_{\varepsilon}^{\alpha} \mathcal{I}^{\alpha}$ are uniformly bounded in $L_{p(\cdot)}$ with respect to $\varepsilon$, that is,

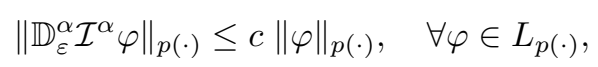

where $c>0$ does not depend on $\varepsilon>0$.

The proof of these two propositions may be found in [1].

We would like to point out that the Riesz derivative $\mathbb{D}^{\alpha}$ does not depend on the order $\ell$ taken in the finite differences. This is why we may omit the parameter $\ell$ in the notation $\mathbb{D}^{\alpha}$. We refer to [24] for details and to [1] where this question was discussed within the setting of the variable Lebesgue spaces.

An important fact concerning hypersingular integrals is their application to the inversion of potential-type operators. There are many papers on this subject, but we only refer to the books [24] and [26], where several references and historical remarks may be found.

The inversion of the Riesz potential operator in the context of the $L_{p(\cdot)}$ spaces was studied in [1], which generalized results from [24] for the case of constant $p$. The inversion theorem from [1] can be formulated as follows.

Theorem 2.5. Let $0<\alpha<n$ and $p(\cdot) \in \mathcal{P}\left(\mathbb{R}^{n}\right)$. Assume also that $\bar{p}<\frac{n}{\alpha}$. Then

$$
\mathbb{D}^{\alpha} \mathcal{I}^{\alpha} \varphi=\varphi, \quad \varphi \in L_{p(\cdot)} .
$$

Moreover, the convergence holds almost everywhere as well, that is,

$$
\lim _{\varepsilon \rightarrow 0} \mathbb{D}_{\varepsilon}^{\alpha} \mathcal{I}^{\alpha} \varphi(x)=\varphi(x),
$$

for almost all $x \in \mathbb{R}^{n}$. 


\section{Characterization of the Riesz potentials on $L_{p(\cdot)}$ spaces}

We define the space of Riesz potentials on $L_{p(\cdot)}$ in a natural way as

$$
\mathcal{I}^{\alpha}\left[L_{p(\cdot)}\right]=\left\{f: f=\mathcal{I}^{\alpha} \varphi, \quad \varphi \in L_{p(\cdot)}\right\}, \quad 0<\alpha<n .
$$

Following approaches in [24], we will show below that the space $\mathcal{I}^{\alpha}\left[L_{p(\cdot)}\right]$ can be described in terms of convergence of hypersingular integrals.

Lemma 3.1. Let $1<\underline{p} \leq p(x) \leq \bar{p}<\frac{n}{\alpha}, x \in \mathbb{R}^{n}$, with $0<\alpha<n$. Let $p^{\prime}(\cdot)$ be the usual conjugate exponent and $q(\cdot)$ be the Sobolev limiting exponent given by $\frac{1}{q(\cdot)}=\frac{1}{p(\cdot)}-\frac{\alpha}{n}$. If $p(\cdot)$ satisfies the logarithmic conditions (2.3) and (2.4), then so do $p^{\prime}(\cdot)$ and $q(\cdot)$.

Proof. The proof is direct.

Theorem 3.2. Let $0<\alpha<n, 1<\underline{p} \leq \bar{p}<\frac{n}{\alpha}$ and let $f$ be $a$ locally integrable function. Assume also that $p(\cdot)$ satisfies the log-Hölder continuity conditions (2.3) and (2.4). Then $f \in \mathcal{I}^{\alpha}\left[L_{p(\cdot)}\right]$, if and only if $f \in L_{q(\cdot)}$, with $\frac{1}{q(\cdot)}=\frac{1}{p(\cdot)}-\frac{\alpha}{n}$, and there exists the Riesz derivative $\mathbb{D}^{\alpha} f$ (in the sense of convergence in $\left.L_{p(\cdot)}\right)$.

Proof. First, assume that $f \in \mathcal{I}^{\alpha}\left[L_{p(\cdot)}\right]$. The fact that $f \in L_{q(\cdot)}$ follows from Theorem 2.2. On the other hand, as $f=\mathcal{I}^{\alpha} \varphi$ for some $\varphi \in L_{p(\cdot)}$, then we have

$$
\mathbb{D}^{\alpha} f=\lim _{\varepsilon \rightarrow 0} \mathbb{D}_{\varepsilon}^{\alpha} \mathcal{I}^{\alpha} \varphi=\varphi
$$

(convergence in $L_{p(\cdot)}$ ) according to Theorem 2.5.

Conversely, let $f \in L_{q(\cdot)}$ and suppose that its Riesz derivative $\mathbb{D}^{\alpha} f$ exists. Our aim is to prove that $f=\mathcal{I}^{\alpha} \mathbb{D}^{\alpha} f$ and hence that $f \in \mathcal{I}^{\alpha}\left[L_{p(\cdot)}\right]$.

Both $f$ and $\mathcal{I}^{\alpha} \mathbb{D}^{\alpha} f$ can be regarded as elements of $\Phi^{\prime}$. Let us show that they coincide in this sense. For all $\phi \in \Phi$, we have:

$$
\begin{aligned}
\int_{\mathbb{R}^{n}} \mathcal{I}^{\alpha} \mathbb{D}^{\alpha} f(x) \phi(x) d x & =\int_{\mathbb{R}^{n}} \mathbb{D}^{\alpha} f(y)\left(\int_{\mathbb{R}^{n}} \frac{\phi(x)}{|x-y|^{n-\alpha}} d x\right) d y \\
& =\lim _{\varepsilon \rightarrow 0} \int_{\mathbb{R}^{n}}\left(\int_{|z|>\varepsilon} \frac{\left(\Delta_{z}^{\ell} f\right)(y)}{d_{n, \ell}(\alpha)|z|^{n+\alpha}} d z\right) \mathcal{I}^{\alpha} \phi(y) d y \\
& =\lim _{\varepsilon \rightarrow 0} \int_{|z|>\varepsilon}\left(\int_{\mathbb{R}^{n}} \frac{\sum_{k=0}^{\ell}(-1)^{k}\left(\begin{array}{l}
\ell \\
k
\end{array}\right) f(u) \mathcal{I}^{\alpha} \phi(u+k z)}{d_{n, \ell}(\alpha)|z|^{n+\alpha}} d u\right) d z
\end{aligned}
$$




$$
\begin{aligned}
& =\lim _{\varepsilon \rightarrow 0} \int_{\mathbb{R}^{n}} f(u)\left(\int_{|z|>\varepsilon} \frac{\left(\Delta_{-z}^{\ell} \mathcal{I}^{\alpha} \phi\right)(u)}{d_{n, \ell}(\alpha)|z|^{n+\alpha}} d z\right) d u \\
& =\lim _{\varepsilon \rightarrow 0} \int_{\mathbb{R}^{n}} f(u) \mathbb{D}_{\varepsilon}^{\alpha} \mathcal{I}^{\alpha} \phi(u) d u \\
& =\int_{\mathbb{R}^{n}} f(u) \phi(u) d u .
\end{aligned}
$$

The first equality follows from the Fubini theorem since the double integral converges absolutely. In fact, since $|\phi(y)| \leq \frac{c}{(1+|y|)^{N}}$ with an arbitrary large $N$, then by Lemma 1.38 in [24], we have that $\mathcal{I}^{\alpha}(|\phi|)(x)$ is bounded and $\mathcal{I}^{\alpha}(|\phi|)(x) \leq \frac{c}{(1+|x|)^{n-\alpha}}$ as $|x| \rightarrow \infty$. Thus

$$
I_{p^{\prime}(\cdot)}\left(\mathcal{I}^{\alpha}(|\phi|)\right) \leq c_{1}+c_{2} \int_{|x|>1} \frac{d x}{(1+|x|)^{(n-\alpha) p^{\prime}(x)}}<\infty
$$

because $\inf _{x \in \mathbb{R}^{n}}(n-\alpha) p^{\prime}(x)>n$. Hence, using Hölder inequality we arrive at

$$
\int_{\mathbb{R}^{n}}\left|\mathbb{D}^{\alpha} f(y)\right| \mathcal{I}^{\alpha}(|\phi|)(y) d y \leq c\left\|\mathbb{D}^{\alpha} f\right\|_{p(\cdot)}\left\|\mathcal{I}^{\alpha}(|\phi|)\right\|_{p^{\prime}(\cdot)}<\infty .
$$

In the second equality, we notice that the convergence with respect to the $L_{p(\cdot)}$ norm implies weak convergence in $\Phi^{\prime}$ (note that $\mathcal{I}^{\alpha} \phi \in \Phi$ ). The third equality follows from similar arguments to those used in the first one, and then by the change of variables $y \rightarrow u+k z$, with fixed $z$. We only observe now that $\mathbb{D}_{\varepsilon}^{\alpha} f \in L_{q(\cdot)}$ (cf. Proposition 2.3 and Lemma 3.1). Finally, the last passage is obtained by making use of the Lebesgue theorem. Indeed, since $\phi \in L_{q^{\prime}(\cdot)}$, then $\mathbb{D}_{\varepsilon}^{\alpha} \mathcal{I}^{\alpha} \phi \in L_{q^{\prime}(\cdot)}$ (cf. Proposition 2.3 and Lemma 3.1), so that $f(\cdot)\left(\mathbb{D}_{\varepsilon}^{\alpha} \mathcal{I}^{\alpha} \phi\right)(\cdot) \in L_{1}$. The result follows now from the inversion Theorem 2.5.

To finish the proof, we observe that since both $f$ and $\mathcal{I}^{\alpha} \mathbb{D}^{\alpha} f$ are tempered distributions, then $\mathcal{I}^{\alpha} \mathbb{D}^{\alpha} f=f+P$, where $P$ is a polynomial. Therefore $f+P \in L_{q(\cdot)}$, which implies $P \in L_{q(\cdot)}$. Thus we should have $P \equiv 0$, which means $\mathcal{I}^{\alpha} \mathbb{D}^{\alpha} f(x)=f(x)$ almost everywhere.

We generalize another characterization which is contained in Theorem 7.11 in [24].

Theorem 3.3. In Theorem 3.2 above one can replace the assertion on the existence of the Riesz derivative of $f$ by the following uniform boundedness condition: there exists $C>0$ such that

$$
\left\|\mathbb{D}_{\varepsilon}^{\alpha} f\right\|_{p(\cdot)} \leq C
$$

for all $\varepsilon>0$. 
Proof. If $f=\mathcal{I}^{\alpha} \varphi, \varphi \in L_{p(\cdot)}$, then (3.2) is immediate by Proposition 2.4.

Conversely, if $\sup _{\varepsilon>0}\left\|\mathbb{D}_{\varepsilon}^{\alpha} f\right\|_{p(\cdot)}<\infty$ then there exists a subsequence of $\left\{\mathbb{D}_{\varepsilon}^{\alpha} f\right\}_{\varepsilon>0}$, say $\left\{\mathbb{D}_{\varepsilon_{k}}^{\alpha} f\right\}_{k \in \mathbb{N}}$, which converges weakly in $L_{p(\cdot)}$ (note that $L_{p(\cdot)}$ is a reflexive Banach space under conditions $1<\underline{p} \leq \bar{p}<\infty$ ). Let us denote its limit by $g \in L_{p(\cdot)}$, and let $\phi \in \Phi$. As in the proof of Theorem 3.2, we get

$$
\begin{aligned}
\int_{\mathbb{R}^{n}} \mathcal{I}^{\alpha} g(x) \phi(x) d x & =\int_{\mathbb{R}^{n}} g(y) \mathcal{I}^{\alpha} \phi(y) d y \\
& =\lim _{k \rightarrow+\infty} \int_{\mathbb{R}^{n}} \mathbb{D}_{\varepsilon_{k}}^{\alpha} f(y) \mathcal{I}^{\alpha} \phi(y) d y \\
& =\lim _{k \rightarrow+\infty} \int_{\mathbb{R}^{n}} f(z)\left(\mathbb{D}_{\varepsilon_{k}}^{\alpha} \mathcal{I}^{\alpha} \phi\right)(z) d z \\
& =\int_{\mathbb{R}^{n}} f(z) \phi(z) d z
\end{aligned}
$$

The second equality follows directly from the weak convergence in $L_{p(\cdot)}$ by noticing that $\mathcal{I}^{\alpha} \phi \in L_{p^{\prime}(\cdot)}$, while the last one is justified by the convergence of $\mathbb{D}_{\varepsilon_{k}}^{\alpha} \mathcal{I}^{\alpha} \phi$ to $\phi$ in $L_{q^{\prime}(\cdot)}$ (taking into account the inversion theorem and the Lemma 3.1 once again) and from the fact that $f \in\left(L_{q^{\prime}(\cdot)}\right)^{\prime}=L_{q(\cdot)}$. Hence, as previously, one arrives at $f=\mathcal{I}^{\alpha} g$, so that $f \in \mathcal{I}^{\alpha}\left[L_{p(\cdot)}\right]$.

\section{Function spaces on $L_{p(\cdot)}$ defined by fractional derivatives}

Hypersingular integrals can also be used to construct function spaces of fractional smoothness. Similarly to the classical case let us consider the space

$$
L_{p(\cdot)}^{\alpha}=\left\{f \in L_{p(\cdot)}: \mathbb{D}^{\alpha} f \in L_{p(\cdot)}\right\}, \quad \alpha>0,
$$

where the fractional derivative $\mathbb{D}^{\alpha}$ is treated in the usual way as convergent in the $L_{p(\cdot)}$ norm. We remark that this space does not depend on the order of the finite differences, and it is a Banach space with respect to the norm

$$
\left\|f \mid L_{p(\cdot)}^{\alpha}\right\|:=\|f\|_{p(\cdot)}+\left\|\mathbb{D}^{\alpha} f\right\|_{p(\cdot)} .
$$

These spaces will be shown the same as the spaces of Bessel potentials. They are connected with the space of Riesz potentials in the following way.

Theorem 4.1. Assume that the exponent $p(\cdot)$ satisfies the usual logarithmic conditions (2.3) and (2.4). Let also $0<\alpha<n$ and $1<\underline{p} \leq$ $\bar{p}<\frac{n}{\alpha}$. 
Then

$$
L_{p(\cdot)}^{\alpha}=L_{p(\cdot)} \cap \mathcal{I}^{\alpha}\left[L_{p(\cdot)}\right] .
$$

Proof. By Theorem 3.2 we only need to prove the embedding $L_{p(\cdot)}^{\alpha} \subset$ $L_{p(\cdot)} \cap \mathcal{I}^{\alpha}\left[L_{p(\cdot)}\right]$. So, let $f \in L_{p(\cdot)}^{\alpha}$. As in the proof of Theorem 3.2 (but here under the assumption that $f \in L_{p(\cdot)}$ instead of $f \in L_{q(\cdot)}$ as there), we have $f(x)=\mathcal{I}^{\alpha} \mathbb{D}^{\alpha} f(x)$ almost everywhere, so that $f \in \mathcal{I}^{\alpha}\left[L_{p(\cdot)}\right]$.

Remark 4.2. Theorem 4.1 also holds if one takes centered differences (everything in the proof of Theorem 3.2 works in a similar way). Hence, from this theorem we conclude that the space $L_{p(\cdot)}^{\alpha}$ does not depend on the type of finite differences used to construct the derivative $\mathbb{D}^{\alpha}$, at least when $\bar{p}<\frac{n}{\alpha}$.

\subsection{Denseness of $C_{0}^{\infty}$ in the space $L_{p(\cdot)}^{\alpha}$}

Before to prove that functions from $L_{p(\cdot)}^{\alpha}$ can be approximated by $C_{0}^{\infty}$ functions, let us show a preliminary denseness result. By $W_{p(\cdot)}^{\infty}$ we denote the Sobolev space of all functions of $L_{p(\cdot)}$ for which all their (weak) derivatives are also in $L_{p(\cdot)}$.

Proposition 4.3. The set $C^{\infty} \cap W_{p(\cdot)}^{\infty}$ is dense in $L_{p(\cdot)}^{\alpha}$ for all $p(\cdot) \in$ $\mathcal{P}\left(\mathbb{R}^{n}\right)$.

Proof. Step 1: Let us show that $C^{\infty} \cap W_{p(\cdot)}^{\infty} \subset L_{p(\cdot)}^{\alpha}$, which is not obvious in the case of variable exponents. If $f \in C^{\infty} \cap W_{p(\cdot)}^{\infty}$ then we already know that

$$
\int_{|y|>\varepsilon} \frac{\left(\Delta_{y}^{\ell} f\right)(x)}{|y|^{n+\alpha}} d y \in L_{p(\cdot)}
$$

for any $\varepsilon>0$ (see Proposition 2.3). On the other hand, we have

$$
\left\|\int_{|y| \leq \delta} \frac{\left(\Delta_{y}^{\ell} f\right)(x)}{|y|^{n+\alpha}} d y\right\|_{p(\cdot)} \rightarrow 0 \quad \text { as } \quad \delta \rightarrow 0 .
$$

To prove (4.2), we use the representation

$$
\begin{aligned}
\left(\Delta_{y}^{\ell} f\right)(x)=r & \sum_{|j|=r} \sum_{k=1}^{\ell} \frac{y^{j}}{j !}(-1)^{r-k} k^{r}\left(\begin{array}{l}
\ell \\
k
\end{array}\right) \\
& \times \int_{0}^{1}(1-t)^{r-1}\left(D^{j} f\right)(x-k t y) d t,
\end{aligned}
$$


(see [24], formula (3.31)) with the choice $\ell \geq r>\alpha$. Hence

$$
\begin{aligned}
& \int_{|y| \leq \delta} \frac{\left(\Delta_{y}^{\ell} f\right)(x)}{|y|^{n+\alpha}} d y \\
& =\sum_{|j|=r} \sum_{k=1}^{\ell} c_{r, j, k} \int_{0}^{1}(1-t)^{r-1}\left(\int_{|y| \leq \delta} \frac{y^{j}}{|y|^{n+\alpha}}\left(D^{j} f\right)(x-k t y) d y\right) d t .
\end{aligned}
$$

The change of variables $y \rightarrow \delta z$ yields

$$
\begin{aligned}
& \int_{|y| \leq \delta} \frac{\left(\Delta_{y}^{\ell} f\right)(x)}{|y|^{n+\alpha}} d y \\
& =\delta^{r-\alpha} \sum_{|j|=r} \sum_{k=1}^{\ell} c_{r, j, k} \int_{0}^{1}(1-t)^{r-1}\left(\frac{1}{\delta_{k}(t)^{n}} K_{j}\left(\frac{\cdot}{\delta_{k}(t)}\right) * D^{j} f\right)(x) d t
\end{aligned}
$$

where $K_{j}$ is given by

$$
K_{j}(z)=\frac{z^{j}}{|z|^{n+\alpha}} \quad \text { when } \quad|z| \leq 1 \quad \text { and } \quad K_{j}(z)=0 \quad \text { otherwise, }
$$

and $\delta_{k}(t)=k \delta t$. Since $|j|=r>\alpha$, the kernel $K_{j}$ has a decreasing radial integrable dominant, so that Theorem 2.1 is applicable and we have

$$
\left|\int_{|y| \leq \delta} \frac{\left(\Delta_{y}^{\ell} f\right)(x)}{|y|^{n+\alpha}} d y\right| \leq \delta^{r-\alpha} \sum_{|j|=r} \sum_{k=1}^{\ell}\left|c_{r, j, k}\right| \int_{0}^{1}(1-t)^{r-1} c \mathcal{M}\left(D^{j} f\right)(x) d t
$$

where $c>0$ is independent of $\delta_{k}(t)$. Hence,

$$
I_{p(\cdot)}\left(\int_{|y| \leq \delta} \frac{\left(\Delta_{y}^{\ell} f\right)(x)}{|y|^{n+\alpha}} d y\right) \leq c \delta^{(r-\alpha) \underline{p}} \sum_{|j|=r} I_{p(\cdot)}\left(\mathcal{M}\left(D^{j} f\right)\right) \rightarrow 0 \text { as } \delta \rightarrow 0 .
$$

We remind that the convergence in norm is equivalent to the modular convergence since the exponent is bounded. Further, under the present assumptions on $p(\cdot)$, the maximal operator $\mathcal{M}$ maps $L_{p(\cdot)}$ into itself.

From (4.1) and (4.5) we see that the integral $\int_{\mathbb{R}^{n}} \frac{\left(\Delta_{y}^{\ell} f\right)(x)}{\mid y^{n+\alpha}} d y$ converges absolutely for all $x$ and defines a function belonging to $L_{p(\cdot)}$. Moreover, by (4.6), it coincides with the Riesz derivative: 


$$
\begin{aligned}
& \| \int_{\mathbb{R}^{n}} \frac{\left(\Delta_{y}^{\ell} f\right)(x)}{|y|^{n+\alpha}} d y-\int_{|y|>\varepsilon} \frac{\left(\Delta_{y}^{\ell} f\right)(x)}{|y|^{n+\alpha}} d y \|_{p(\cdot)} \\
&=\left\|\int_{|y| \leq \varepsilon} \frac{\left(\Delta_{y}^{\ell} f\right)(x)}{|y|^{n+\alpha}} d y\right\|_{p(\cdot)} \rightarrow 0 \quad \text { as } \quad \varepsilon \rightarrow 0,
\end{aligned}
$$

so that $\mathbb{D}^{\alpha} f \in L_{p(\cdot)}$.

We remark that some modification is needed if $\alpha$ is odd. In this case, we should consider centered differences (and hence $\ell>\alpha$; recall the previous rule) and then proceed in a similar way from (4.3).

Step 2: We use the standard approximation by using mollifiers (as in [24], Lemma 7.14). Let $\varphi \in C_{0}^{\infty}$ such that $\varphi \geq 0, \int_{\mathbb{R}^{n}} \varphi(x) d x=1$ with $\operatorname{supp} \varphi \subset \overline{B(0,1)}$. Put $\varphi_{m}(x):=m^{n} \varphi(m x), m \in \mathbb{N}$. Then $\varphi_{m} \in C_{0}^{\infty}$ and supp $\varphi_{m} \subset \overline{B(0,1 / m)}$. Given $f \in L_{p(\cdot)}^{\alpha}$ let us define

$$
f_{m}(x):=\varphi_{m} * f(x)=\int_{\mathbb{R}^{n}} \varphi(y) f\left(x-\frac{y}{m}\right) d y .
$$

Hence $f_{m} \in C^{\infty}$. We also have $f_{m} \in L_{p(\cdot)}$ by Theorem 2.1 and $D^{j} f_{m}=D^{j}\left(\varphi_{m}\right) * f \in L_{p(\cdot)}$. In the case of fractional derivatives we have, for each $\varepsilon>0$ and $m \in \mathbb{N}$,

$$
\mathbb{D}_{\varepsilon}^{\alpha} f_{m}=\left(\mathbb{D}_{\varepsilon}^{\alpha} f\right)_{m}
$$

that is,

$$
\mathbb{D}_{\varepsilon}^{\alpha}\left(\varphi_{m} * f\right)=\varphi_{m} * \mathbb{D}_{\varepsilon}^{\alpha} f
$$

which can be easily proved by Fubini theorem. Hence

$$
\mathbb{D}^{\alpha} f_{m}=\lim _{\varepsilon \rightarrow 0}\left(\varphi_{m} * \mathbb{D}_{\varepsilon}^{\alpha} f\right)=\varphi_{m} * \mathbb{D}^{\alpha} f=\left(\mathbb{D}^{\alpha} f\right)_{m},
$$

where the second equality follows from the continuity of the convolution operator. In particular one concludes that $\mathbb{D}^{\alpha} f_{m} \in L_{p(\cdot)}$.

It remains to show that the functions $f_{m}$ approximate the function $f$ in the $L_{p(\cdot)}^{\alpha}$ norm. Of course, $\left\|f-f_{m}\right\|_{p(\cdot)} \rightarrow 0$ as $m \rightarrow \infty$ by Theorem 2.1 once again. On the other hand, using similar arguments we have

$$
\left\|\mathbb{D}^{\alpha}\left(f-f_{m}\right)\right\|_{p(\cdot)}=\left\|\mathbb{D}^{\alpha} f-\mathbb{D}^{\alpha} f_{m}\right\|_{p(\cdot)}=\left\|\mathbb{D}^{\alpha} f-\left(\mathbb{D}^{\alpha} f\right)_{m}\right\|_{p(\cdot)} \rightarrow 0
$$

as $m \rightarrow \infty$, since $\mathbb{D}^{\alpha} f \in L_{p(\cdot)}$.

Theorem 4.4. If $p(\cdot)$ is as in Proposition 4.3 with $\bar{p}<\frac{n}{\alpha}$, then the class $C_{0}^{\infty}$ is dense in $L_{p(\cdot)}^{\alpha}$. 
Proof. By Proposition 4.3, it is sufficient to show that every function $f \in C^{\infty} \cap W_{p(\cdot)}^{\infty}$ can be approximated by functions in $C_{0}^{\infty}$ in the norm $\left\|\cdot \mid L_{p(\cdot)}^{\alpha}\right\|$. As in the case of constant $p$, we will use the "smooth truncation" of functions.

Let $\mu \in C_{0}^{\infty}$ with $\mu(x)=1$ if $|x| \leq 1, \operatorname{supp} \mu \subset \overline{B(0,2)}$ and $0 \leq \mu(x) \leq 1$ for every $x$. Define $\mu_{m}(x):=\mu\left(\frac{x}{m}\right), x \in \mathbb{R}^{n}, m \in \mathbb{N}$. We are to show that the sequence of truncations $\left\{\mu_{m} f\right\}_{m \in \mathbb{N}}$ converges to $f$ in $L_{p(\cdot)}^{\alpha}$.

The passage to the limit $\lim _{m \rightarrow \infty}\left\|f-\mu_{m} f\right\|_{p(\cdot)}=0 \Longleftrightarrow \lim _{m \rightarrow \infty} I_{p(\cdot)}(f-$ $\left.\mu_{m} f\right)=0$ is directly checked by means of the Lebesgue dominated convergence theorem. It remains to show that $I_{p(\cdot)}\left(\mathbb{D}^{\alpha}\left(f-\mu_{m} f\right)\right)$ also tends to zero as $m \rightarrow \infty$.

Taking Remark 4.2 into account, we may consider centered differences in the fractional derivative (under the choice $\ell>\alpha$ with $\ell$ even). For brevity we denote $\nu_{m}=1-\mu_{m}$. Then we have

$$
\begin{aligned}
\mathbb{D}^{\alpha}\left(\nu_{m} f\right)(x) & =\frac{1}{d_{n, \ell}(\alpha)} \sum_{k=0}^{\ell}\left(\begin{array}{l}
\ell \\
k
\end{array}\right) \int_{\mathbb{R}^{n}} \frac{\left(\Delta_{y}^{k} \nu_{m}\right)\left(x+\frac{\ell}{2} y\right)\left(\Delta_{y}^{\ell-k} f\right)\left(x+\left(\frac{\ell}{2}-k\right) y\right)}{|y|^{n+\alpha}} d y \\
& =: \frac{1}{d_{n, \ell}(\alpha)} \sum_{k=0}^{\ell}\left(\begin{array}{l}
\ell \\
k
\end{array}\right) A_{m, k} f(x) .
\end{aligned}
$$

So we need to show that $I_{p(\cdot)}\left(A_{m, k} f\right) \rightarrow 0$ as $m \rightarrow \infty$, for $k=0,1, \ldots, \ell$. We separately treat the cases $k=0, k=\ell$ and $1 \leq k \leq \ell-1$.

The case $k=0$ : we have

$$
A_{m, 0} f(x)=d_{n, \ell}(\alpha) \nu_{m}(x) \mathbb{D}^{\alpha} f(x)+B_{m} f(x)
$$

where

$$
\begin{aligned}
B_{m} f(x) & =\int_{\mathbb{R}^{n}} \frac{\left[\nu_{m}\left(x+\frac{\ell}{2} y\right)-\nu_{m}(x)\right]\left(\Delta_{y}^{\ell} f\right)\left(x+\frac{\ell}{2} y\right)}{|y|^{n+\alpha}} d y \\
& =\int_{\mathbb{R}^{n}} \frac{\left[\mu_{m}(x)-\mu_{m}\left(x+\frac{\ell}{2} y\right)\right]\left(\Delta_{y}^{\ell} f\right)\left(x+\frac{\ell}{2} y\right)}{|y|^{n+\alpha}} d y
\end{aligned}
$$

The convergence of the first term in (4.7) is clear, so that it remains to prove that $I_{p(\cdot)}\left(B_{m} f\right) \rightarrow 0$ as $m \rightarrow \infty$. Put

$$
B_{m} f(x)=\int_{|y| \leq 1}(\cdots) d y+\int_{|y|>1}(\cdots) d y:=B_{m}^{0} f(x)+B_{m}^{1} f(x) .
$$

To estimate the term $B_{m}^{0} f$, we make use of the Taylor formula (of order 1) with the remainder in the integral form and obtain 


$$
\mu_{m}\left(x+\frac{\ell}{2} y\right)-\mu_{m}(x)=\frac{\ell}{2 m} \sum_{j=1}^{n} y_{j} \int_{0}^{1} \frac{\partial \mu}{\partial x_{j}}\left(\frac{x+\frac{\ell t}{2} y}{m}\right) d t .
$$

Hence

$$
\left|\mu_{m}\left(x+\frac{\ell}{2} y\right)-\mu_{m}(x)\right| \leq \frac{c}{m}|y|
$$

where $c>0$ does not depend on $x, y$ and $m$.

As in the proof of Proposition 4.3, we can estimate $B_{m}^{0} f$ in terms of the convolution of the derivatives of $f$ with a "good kernel" in the sense of Theorem 2.1. In fact, taking (4.8) and (3.31) in [24] into account, we get

$$
\begin{aligned}
& \left|B_{m}^{0} f(x)\right| \\
& \leq \quad \frac{c}{m} \sum_{|j|=r} \sum_{\nu=\frac{\ell}{2}}^{\ell}\left[\int_{0}^{\frac{\ell}{2 \nu}}(1-t)^{r-1}\left(\frac{1}{\left(-\theta_{\nu}(t)\right)^{n}} K\left(\frac{\cdot}{-\theta_{\nu}(t)}\right) *\left|D^{j} f\right|\right)(x) d t\right. \\
& \left.\quad+\int_{\frac{\ell}{2 \nu}}^{1}(1-t)^{r-1}\left(\frac{1}{\theta_{\nu}(t)^{n}} K\left(\frac{\cdot}{\theta_{\nu}(t)}\right) *\left|D^{j} f\right|\right)(x) d t\right] \\
& (4.9) \quad+\frac{c}{m} \sum_{|j|=r} \sum_{\nu=1}^{\frac{\ell}{2}-1} \int_{0}^{1}(1-t)^{r-1}\left(\frac{1}{\left(-\theta_{\nu}(t)\right)^{n}} K\left(\frac{\cdot}{-\theta_{\nu}(t)}\right) *\left|D^{j} f\right|\right)(x) d t
\end{aligned}
$$

where $K$ is given by

$$
K(z)=|z|^{r+1-n-\alpha} \quad \text { if } \quad|z|<1 \quad \text { and } \quad K(z)=0 \quad \text { otherwise, }
$$

with $\theta_{\nu}(t)=\nu t-\frac{\ell}{2}$ and under the choice $r>\alpha-1$. So

$$
I_{p(\cdot)}\left(B_{m}^{0} f\right) \leq \frac{c}{m} \sum_{|j|=r} I_{p(\cdot)}\left[\mathcal{M}\left(\left|D^{j} f\right|\right)\right] \rightarrow 0 \quad \text { as } m \rightarrow \infty .
$$

For the term $B_{m}^{1} f$ we may proceed as follows. Since $\mu$ is infinitely differentiable and compactly supported, then it satisfies the Hölder continuity condition. Hence, for an arbitrary $\varepsilon \in(0,1]$, there exists $c=c_{\varepsilon}>0$ not depending on $x, y$, such that

$$
\left|\mu_{m}\left(x+\frac{\ell}{2} y\right)-\mu_{m}(x)\right| \leq \frac{c}{m^{\varepsilon}}|y|^{\varepsilon} .
$$

When $\alpha>1$, we may proceed as before by considering $r<\alpha<\ell$. Putting all these things together, one estimates $B_{m}^{1} f(x)$ as in (4.9) with 
the correspondent kernel $K$ given by

$$
K(y)=\frac{|y|^{r}}{|y|^{n+\alpha-\varepsilon}} \quad \text { when } \quad|y|>1 \quad \text { and } \quad K(y)=0 \quad \text { otherwise. }
$$

Under the choice $0<\varepsilon<\min (1, \alpha-r)$, the kernel $K$ has an integrable radial decreasing dominant, so that we can apply Stein's theorem once more and arrive at the conclusion that

$$
I_{p(\cdot)}\left(B_{m}^{1} f\right) \leq \frac{c}{m^{\varepsilon}} \sum_{|j|=r} I_{p(\cdot)}\left(\left|D^{j} f\right|\right) \rightarrow 0 \quad \text { as } \quad m \rightarrow \infty .
$$

The case $0<\alpha \leq 1$ can be treated without passing to the derivatives of $f$. In fact, in this case, we may take $\ell=2$, and hence

$$
\left|B_{m}^{1} f(x)\right| \leq \frac{c}{m^{\varepsilon}}\left(\int_{|y|>1} \frac{|f(x+y)|}{|y|^{n+\alpha-\varepsilon}} d y+\int_{|y|>1} \frac{|f(x)|}{|y|^{n+\alpha-\varepsilon}} d y+\int_{|y|>1} \frac{|f(x-y)|}{|y|^{n+\alpha-\varepsilon}} d y\right) .
$$

Each term can be managed by using similar arguments as above but now with the choice $0<\varepsilon<\alpha$.

$\underline{\text { The case } k=\ell \text { : let }}$

$$
\begin{aligned}
A_{m, \ell} f(x) & =\int_{\mathbb{R}^{n}} \frac{\left(\Delta_{y}^{\ell} \nu_{m}\right)\left(x+\frac{\ell}{2} y\right) f\left(x-\frac{\ell}{2} y\right)}{|y|^{n+\alpha}} d y \\
& =\int_{|y| \leq 1}(\cdots) d y+\int_{|y|>1}(\cdots) d y \\
& =: \quad B_{m, \ell}^{0} f(x)+B_{m, \ell}^{1} f(x)
\end{aligned}
$$

Notice that $\left(\Delta_{y}^{\ell} \nu_{m}\right)(z)=-\left(\Delta_{y}^{\ell} \mu_{m}\right)(z)=-\left(\Delta_{\frac{y}{m}}^{\ell} \mu\right)\left(\frac{z}{m}\right)$. So, according to (4.3), one gets the estimate

$$
\begin{aligned}
\left|\left(\Delta_{y}^{\ell} \nu_{m}\right)\left(x+\frac{\ell}{2} y\right)\right| & =\left|\left(\Delta_{\frac{y}{m}}^{\ell} \mu\right)\left(\frac{x+\frac{\ell}{2} y}{m}\right)\right| \\
& \leq c\left(\frac{|y|}{m}\right)^{r} \sum_{|j|=r}\left\|D^{j} \mu\right\|_{\infty} \\
& \leq \frac{c}{m^{r}}|y|^{r}
\end{aligned}
$$

(with $\ell \geq r>\alpha$ ). Hence,

$$
\left|B_{m, \ell}^{0} f(x)\right| \leq \frac{c}{m^{r}}(K *|f|)(x)
$$


where $K$ is now given by

$$
K(y)=\frac{1}{|y|^{n+\alpha-r}} \quad \text { if } \quad|y| \leq \frac{\ell}{2} \quad \text { and } \quad K(y)=0 \quad \text { otherwise. }
$$

Since $r>\alpha$, the kernel $K$ is under the assumptions of Theorem 2.1. As before, we get $\left\|B_{m, \ell}^{0} f\right\|_{p(\cdot)} \rightarrow 0$ as $m \rightarrow \infty$.

As far as the term $B_{m, \ell}^{1} f$ is concerned, when $\alpha>1$ we may choose $\ell>\alpha>r$ and proceed in a similar way as in the case $k=0$ above. When $0<\alpha \leq 1$ we may take $\ell=2$ and get

$$
\begin{aligned}
\left|B_{m, \ell}^{1} f(x)\right| \leq & \int_{|y|>1} \frac{\left|\left(\Delta_{\frac{y}{m}}^{2} \mu\right)\left(\frac{x+y}{m}\right)\right||f(x-y)|}{|y|^{n+\alpha}} d y \\
= & \int_{|y|>1} \frac{\left|\mu\left(\frac{x+y}{m}\right)-2 \mu\left(\frac{x}{m}\right)+\mu\left(\frac{x-y}{m}\right)\right||f(x-y)|}{|y|^{n+\alpha}} d y \\
\leq & \int_{|y|>1} \frac{\left|\mu\left(\frac{x+y}{m}\right)-\mu\left(\frac{x}{m}\right)\right||f(x-y)|}{|y|^{n+\alpha}} d y \\
& +\int_{|y|>1} \frac{\left|\mu\left(\frac{x-y}{m}\right)-\mu\left(\frac{x}{m}\right)\right||f(x-y)|}{|y|^{n+\alpha}} d y \\
\leq & \frac{c}{m^{\varepsilon}} \int_{|y|>1} \frac{|y|^{\varepsilon}|f(x-y)|}{|y|^{n+\alpha}} d y
\end{aligned}
$$

for any $\varepsilon \in(0,1]$ (and $c>0$ independent of $m$ ). Thus we arrive at the desired conclusion by taking $\varepsilon<\alpha$.

The case $k \in\{1,2, \ldots, \ell-1\}$ : as in the previous case, we have

$$
\begin{aligned}
A_{m, k} f(x) & =\int_{\mathbb{R}^{n}} \frac{\left(\Delta_{y}^{k} \nu_{m}\right)\left(x+\frac{\ell}{2} y\right)\left(\Delta_{y}^{\ell-k} f\right)\left(x+\left(\frac{\ell}{2}-k\right) y\right)}{|y|^{n+\alpha}} d y \\
& =\int_{|y| \leq 1}(\cdots) d y+\int_{|y|>1}(\cdots) d y \\
& =: \quad B_{m, k}^{0} f(x)+B_{m, k}^{1} f(x) .
\end{aligned}
$$

We may estimate the term $B_{m, k}^{0} f$ by noticing that

$$
\left|\left(\Delta_{y}^{k} \nu_{m}\right)\left(x+\frac{\ell}{2} y\right)\right| \leq c\left(\frac{|y|}{m}\right)^{k}
$$

and then by proceeding as above with an appropriate choice of $r$. 
For the term $B_{m, k}^{1}$ we first consider the case $\alpha>1$. Since $\left(\begin{array}{l}k \\ l\end{array}\right)=\left(\begin{array}{c}k \\ k-l\end{array}\right)$, for $l=0,1, \ldots, k$, we may write

$$
\left(\Delta_{\frac{y}{m}}^{k} \mu\right)\left(\frac{x+\frac{\ell}{2} y}{m}\right)=\sum_{l=0}^{\frac{k-1}{2}}\left(\begin{array}{l}
k \\
l
\end{array}\right)\left[\mu\left(\frac{x+\frac{\ell}{2} y}{m}-l \frac{y}{m}\right)-\mu\left(\frac{x+\frac{\ell}{2} y}{m}-(k-l) \frac{y}{m}\right)\right]
$$

if $k$ is odd. When $k$ is even, we can also represent our finite difference as the sum of the first order differences of two appropriate terms since $\sum_{l=0}^{k}(-1)^{l}\left(\begin{array}{l}k \\ l\end{array}\right)=0$. In both situations we may again make use of the Hölder continuity (of order $\varepsilon$ ) of the function $\mu$. Finally, we shall arrive at the desired estimate by using arguments as above, but under the assumption $0<\varepsilon<\min (1, \alpha-1)$. The case $0<\alpha \leq 1$ can be easily solved by taking $\ell=2$. So, we have $k=\ell-k=1$ and hence

$$
\begin{aligned}
\left|B_{m, k}^{1} f(x)\right| & \leq \int_{|y|>1} \frac{\left|\left(\Delta_{\frac{y}{m}}^{1} \mu\right)\left(\frac{x+y}{m}\right)\right|\left|\left(\Delta_{y}^{1} f\right)(x)\right|}{|y|^{n+\alpha}} d y \\
& \leq \int_{|y|>1} \frac{\left(\frac{|y|}{m}\right)^{\varepsilon}|f(x)|}{|y|^{n+\alpha}} d y+\int_{|y|>1} \frac{\left(\frac{|y|}{m}\right)^{\varepsilon}|f(x-y)|}{|y|^{n+\alpha}} d y,
\end{aligned}
$$

so that we can proceed as in the previous cases.

\section{Bessel potentials on $L_{p}(\cdot)$ spaces and their characterization}

The main aim of this section is to describe the range of the Bessel potential operator on $L_{p(\cdot)}$ in terms of convergence of hypersingular integrals. This is known in the case of constant $p$, see [24], Section 7.2, or [26], Section 27.3 , and references therein. Here we consider the case $0<\alpha<n, \bar{p}<\frac{n}{\alpha}$.

5.1 Basic properties. The Bessel kernel $G_{\alpha}$ can be introduced in terms of Fourier transform by

$$
\widehat{G}_{\alpha}(x)=\left(1+|x|^{2}\right)^{-\alpha / 2}, \quad x \in \mathbb{R}^{n}, \quad \alpha>0 .
$$

It is known that

$$
G_{\alpha}(x)=c(\alpha) \int_{0}^{\infty} e^{-\frac{\pi|x|^{2}}{t}-\frac{t}{4 \pi}} t^{\frac{\alpha-n}{2}} \frac{d t}{t}, \quad x \in \mathbb{R}^{n},
$$

where $c(\alpha)$ is a certain constant (see, for example, [29], Section V.3.1), so that $G_{\alpha}$ is a non-negative, radially decreasing function. Moreover, $G_{\alpha}$ 
is integrable with $\left\|G_{\alpha}\right\|_{1}=\widehat{G}_{\alpha}(0)=1$ and it can also be represented by means of the McDonald function:

$$
G_{\alpha}(x)=c(\alpha, n)|x|^{\frac{\alpha-n}{2}} K_{\frac{n-\alpha}{2}}(|x|) .
$$

The Bessel potential of order $\alpha>0$ of the density $\varphi$ is defined by

$$
\mathcal{B}^{\alpha} \varphi(x)=\int_{\mathbb{R}^{n}} G_{\alpha}(x-y) \varphi(y) d y .
$$

For convenience, we also denote $\mathcal{B}^{0} \varphi=\varphi$.

Theorem 5.1. If $p(\cdot) \in \mathcal{P}\left(\mathbb{R}^{n}\right)$ then the Bessel potential operator $\mathcal{B}^{\alpha}$ is bounded in $L_{p(\cdot)}$.

Proof. The boundedness of the operator $\mathcal{B}^{\alpha}$ follows from the properties of the kernel $G_{\alpha}$ described above. Taking into account Theorem 2.1, there exists a constant $c>0$ such that

$$
\left\|\mathcal{B}^{\alpha} \varphi\right\|_{p(\cdot)}=\left\|G_{\alpha} * \varphi\right\|_{p(\cdot)} \leq c\|\varphi\|_{p(\cdot)}, \text { for all } \varphi \in L_{p(\cdot)} .
$$

We are interested in Bessel potentials with densities in $L_{p(\cdot)}$. One defines the space of Bessel potentials as the range of the Bessel potential operator

$$
\mathcal{B}^{\alpha}\left[L_{p(\cdot)}\right]=\left\{f: f=\mathcal{B}^{\alpha} \varphi, \quad \varphi \in L_{p(\cdot)}\right\}, \quad \alpha \geq 0 .
$$

According to Theorem 5.1, the space $\mathcal{B}^{\alpha}\left[L_{p(\cdot)}\right]$, also called sometimes Liouville space of fractional smoothness, is well defined, being a subspace of $L_{p(\cdot)}$ if the maximal operator is bounded in $L_{p(\cdot)}$, in particular, if the exponent $p(\cdot)$ is $\log$-Hölder continuous both locally and at infinity.

$\mathcal{B}^{\alpha}\left[L_{p(\cdot)}\right]$ is a Banach space endowed with the norm

$$
\left\|f \mid \mathcal{B}^{\alpha}\left[L_{p(\cdot)}\right]\right\|:=\|\varphi\|_{p(\cdot)},
$$

where $\varphi$ is the density from (5.1).

The symbol" $\hookrightarrow$ ” below denotes continuous embedding.

Proposition 5.2. If $p(\cdot) \in \mathcal{P}\left(\mathbb{R}^{n}\right)$ and $\alpha>\gamma \geq 0$, then $\mathcal{B}^{\alpha}\left[L_{p(\cdot)}\right] \hookrightarrow$ $\mathcal{B}^{\gamma}\left[L_{p(\cdot)}\right]$.

Proof. The proof follows immediately from the properties of the Bessel kernel and from the boundedness of the Bessel potential operator. Indeed, if $f=\mathcal{B}^{\alpha} \varphi$ for some $\varphi \in L_{p(\cdot)}$ then one can write $f=\mathcal{B}^{\gamma}\left(\mathcal{B}^{\alpha-\gamma} \varphi\right)$. Thus $f \in \mathcal{B}^{\gamma}\left[L_{p(\cdot)}\right]$ by Theorem 5.1. Furthermore,

$$
\left\|f\left|\mathcal{B}^{\gamma}\left[L_{p(\cdot)}\right]\|:=\| \mathcal{B}^{\alpha-\gamma} \varphi\left\|_{p(\cdot)} \leq c\right\| \varphi\left\|_{p(\cdot)}=: c\right\| f\right| \mathcal{B}^{\alpha}\left[L_{p(\cdot)}\right]\right\| .
$$


5.2 Characterization of the space $B^{\alpha}\left[L_{p(\cdot)}\right]$ via hypersingular integrals. The comparison of the ranges of the Bessel and Riesz potential operators is naturally made via the convolution type operator whose symbol is the ratio of the Fourier transforms of the Riesz and Bessel kernels. This operator is the sum of the identity operator and the convolution operator with a radial integrable kernel. Keeping in mind the application of Theorem 2.1 , we have to show more, namely that this kernel has an integrable decreasing dominant.

We have to show the existence of integrable decreasing dominants for two important kernels $g_{\alpha}$ and $h_{\alpha}$, one defined in (5.3), another in (5.4). This will require substantial efforts.

Let $g_{\alpha}$ and $h_{\alpha}$ be the functions defined via the following Fourier transforms

$$
\begin{aligned}
& \frac{|x|^{\alpha}}{\left(1+|x|^{2}\right)^{\frac{\alpha}{2}}}=1+\widehat{g}_{\alpha}(x), \quad \alpha>0, \quad x \in \mathbb{R}^{n}, \\
& \frac{\left(1+|x|^{2}\right)^{\frac{\alpha}{2}}}{1+|x|^{\alpha}}=1+\widehat{h}_{\alpha}(x), \quad \alpha>0, \quad x \in \mathbb{R}^{n} .
\end{aligned}
$$

Observe that

$$
\frac{1+|x|^{\alpha}}{\left(1+|x|^{2}\right)^{\frac{\alpha}{2}}}=\widehat{G}_{\alpha}(x)+\widehat{g}_{\alpha}(x)+1 .
$$

It is known that $g_{\alpha}$ and $h_{\alpha}$ are integrable (see, for example, Lemma 1.25 in [24]).

The following two lemmas are crucial for our further goals.

Lemma 5.3. The function $g_{\alpha}$ defined in (5.3) has an integrable and radially decreasing dominant.

Lemma 5.4. The kernel $h_{\alpha}$ given by (5.4) admits the bounds

$$
\left|h_{\alpha}(x)\right| \leq \frac{c}{|x|^{n-a}} \quad \text { as } \quad|x|<1, \quad a=\min \{1, \alpha\} \text {, }
$$

and

$$
\left|h_{\alpha}(x)\right| \leq \frac{c}{|x|^{n+\alpha}} \quad \text { as } \quad|x| \geq 1,
$$

where $c>0$ is a constant not depending on $x$.

The proof of these lemmas being somewhat technical is postponed till the next subsection. 
Before to formulate the main result of this section, we prove the following two statements.

Proposition 5.5. Let $0<\alpha<n$ and $p(\cdot) \in \mathcal{P}\left(\mathbb{R}^{n}\right)$ with $1<\underline{p} \leq \bar{p}<$ $n / \alpha$. Then every $\varphi \in L_{p(\cdot)}^{\alpha}$ can be represented as

$$
\varphi=\mathcal{B}^{\alpha}\left(I+U_{\alpha}\right)\left(\varphi+\mathbb{D}^{\alpha} \varphi\right),
$$

where $I$ denotes the identity operator and $U_{\alpha}$ is the convolution operator with the kernel $h_{\alpha}$.

Proof. Identity (5.8) holds for functions $\varphi \in C_{0}^{\infty}$. This follows immediately from equality (5.4) above (cf. (7.39) in [24]). The denseness of $C_{0}^{\infty}$ in $L_{p(\cdot)}^{\alpha}$ (stated in Theorem 4.4) allows us to write (5.8) for all functions in $L_{p(\cdot)}^{\alpha}$. To this end, we observe that both operators $\mathcal{B}^{\alpha}$ and $U_{\alpha}$ are continuous in $L_{p(\cdot)}$. In fact, the boundedness of $\mathcal{B}^{\alpha}$ was proved in Theorem 5.1. On the other hand, the convolution operator $U_{\alpha}$ is bounded since its kernel has a radially decreasing and integrable dominant by Lemma 5.4.

Proposition 5.6. Let $0<\alpha<n$ and let $1<\underline{p} \leq \bar{p}<n / \alpha$. Then

$$
\mathcal{B}^{\alpha} \psi=\mathcal{I}^{\alpha}\left(I+K_{\alpha}\right) \psi
$$

for all $\psi \in L_{\underline{p}}+L_{\bar{p}}$, where $I$ is the identity operator and $K_{\alpha}$ is the convolution operator with the kernel $g_{\alpha}$.

Proof. Representation (5.9) holds for densities belonging to classical Lebesgue spaces (see, for instance, (7.38) in [24]), where the kernel of $K_{\alpha}$ is precisely the function $g_{\alpha}$ from (5.3). By the Sobolev theorem one concludes that either $\mathcal{B}$ or $\mathcal{I}^{\alpha}\left(I+K_{\alpha}\right)$ are linear operators from $L_{\underline{p}}$ into $L_{q(\underline{p})}$, with $\frac{1}{q(\underline{p})}=\frac{1}{p}-\frac{\alpha}{n}$, and from $L_{\bar{p}}$ into $L_{q(\bar{p})}$, with $\frac{1}{q(\bar{p})}=\frac{1}{\bar{p}}-\frac{\alpha}{n}$. So, we can define these operators on the sum $L_{p}+L_{\bar{p}}$ in the usual way. Hence, if $\psi=\psi_{0}+\psi_{1}$, with $\psi_{0} \in L_{\underline{p}}$ and $\psi_{1}^{-} \in L_{\bar{p}}$, then we may make use the already known representation for each term and then arrive at equality (5.9).

Finally, we are able to characterize the Bessel potentials in terms of convergence of hypersingular integrals. The following theorem in the case of constant $p, 1<p<\infty$, is due to E. Stein [28] when $0<\alpha<1$ and to P.I. Lizorkin [17] in the general case $0<\alpha<\infty$, see also the proof for constant $p$ in [24], p.186.

Theorem 5.7. Let $0<\alpha<n$. If $1<p \leq \bar{p}<n / \alpha$ and $p(\cdot)$ satisfies the $\log$-Hölder continuity conditions (2.3) and (2.4), then $\mathcal{B}^{\alpha}\left[L_{p(\cdot)}\right]=L_{p(\cdot)}^{\alpha}$ 
with equivalent norms: there are constants $c_{1}, c_{2}>0$ such that

$$
c_{1}\left\|f\left|L_{p(\cdot)}^{\alpha}\|\leq\| f\right| \mathcal{B}^{\alpha}\left[L_{p(\cdot)}\right]\right\| \leq c_{2}\left\|f \mid L_{p(\cdot)}^{\alpha}\right\|, \quad \forall f \in \mathcal{B}^{\alpha}\left[L_{p(\cdot)}\right] .
$$

Proof. Assume first that $f \in \mathcal{B}^{\alpha}\left[L_{p(\cdot)}\right]$. Then $f \in L_{p(\cdot)}$ by Theorem 5.1. It remains to show that its Riesz derivative also belongs to $L_{p(\cdot)}$. Since $f=\mathcal{B}^{\alpha} \varphi$ for some $\varphi \in L_{p(\cdot)}$ and $L_{p(\cdot)} \subset L_{\underline{p}}+L_{\bar{p}}$, then by Proposition 5.6 one gets the representation

$$
\mathcal{B}^{\alpha} \varphi=\mathcal{I}^{\alpha}\left(I+K_{\alpha}\right) \varphi .
$$

Lemma 5.3 combined with Theorem 2.1, allow us to conclude that $K_{\alpha}$ is bounded in $L_{p(\cdot)}$, and hence $f \in \mathcal{I}^{\alpha}\left[L_{p(\cdot)}\right]$. So, according to the characterization given in Theorem 3.2, the Riesz derivative $\mathbb{D}^{\alpha} f$ exists in the sense of convergence in $L_{p(\cdot)}$. Therefore, $f \in L_{p(\cdot)}^{\alpha}$. Moreover,

$$
\begin{aligned}
\left\|f \mid L_{p(\cdot)}^{\alpha}\right\| & =\left\|\mathcal{B}^{\alpha} \varphi\right\|_{p(\cdot)}+\left\|\mathbb{D}^{\alpha} \mathcal{B}^{\alpha} \varphi\right\|_{p(\cdot)} \\
& =\left\|\mathcal{B}^{\alpha} \varphi\right\|_{p(\cdot)}+\left\|\mathbb{D}^{\alpha} \mathcal{I}^{\alpha}\left(I+K_{\alpha}\right) \varphi\right\|_{p(\cdot)} \\
& =\left\|\mathcal{B}^{\alpha} \varphi\right\|_{p(\cdot)}+\left\|\left(I+K_{\alpha}\right) \varphi\right\|_{p(\cdot)} \\
& \leq c\|\varphi\|_{p(\cdot)}=c\left\|f \mid \mathcal{B}^{\alpha}\left[L_{p(\cdot)}^{\alpha}\right]\right\| .
\end{aligned}
$$

The third equality follows from the inversion Theorem 2.5, while the inequality is obtained from Theorem 5.1 and from the boundedness of $K_{\alpha}$.

Conversely, suppose that $f \in L_{p(\cdot)}^{\alpha}$. Proposition 5.5 yields the representation

$$
f=\mathcal{B}^{\alpha}\left(I+U_{\alpha}\right)\left(f+\mathbb{D}^{\alpha} f\right) .
$$

Taking into account Lemma 5.4 and Theorem 2.1, we arrive at the conclusion that $f \in \mathcal{B}^{\alpha}\left[L_{p(\cdot)}\right]$ and

$$
\begin{aligned}
\left\|f \mid \mathcal{B}^{\alpha}\left[L_{p(\cdot)}\right]\right\| & =\left\|\left(I+U_{\alpha}\right)\left(f+\mathbb{D}^{\alpha} f\right)\right\|_{p(\cdot)} \\
& \leq c\left(\|f\|_{p(\cdot)}+\left\|\mathbb{D}^{\alpha} f\right\|_{p(\cdot)}\right)=c\left\|f \mid L_{p(\cdot)}^{\alpha}\right\| .
\end{aligned}
$$

Corollary 5.8. If the exponent $p(\cdot)$ is under the conditions of Theorem 5.7, then $C_{0}^{\infty}$ is dense in $\mathcal{B}^{\alpha}\left[L_{p(\cdot)}\right]$.

5.3 Proof of Lemmas $\mathbf{5 . 3}$ and 5.4. We start by proving Lemma 5.3. Let us denote $\rho=\left(1+|x|^{2}\right)^{1 / 2}$. Then $\frac{|x|^{\alpha}}{\left(1+|x|^{2}\right)^{\frac{\alpha}{2}}}-1=\left(1-\rho^{-2}\right)^{\alpha / 2}-1$. Taking the expansion into the binomial series we get

$$
\left(1-\rho^{-2}\right)^{\frac{\alpha}{2}}-1=\sum_{k=0}^{\infty}\left(\begin{array}{c}
\alpha / 2 \\
k
\end{array}\right)\left(-\rho^{-2}\right)^{k}-1=\sum_{k=1}^{\infty}(-1)^{k}\left(\begin{array}{c}
\alpha / 2 \\
k
\end{array}\right) \rho^{-2 k}, \quad \rho>1 .
$$


Hence, for each $x \neq 0$,

$$
\frac{|x|^{\alpha}}{\left(1+|x|^{2}\right)^{\frac{\alpha}{2}}}-1=\sum_{k=1}^{\infty}(-1)^{k}\left(\begin{array}{c}
\alpha / 2 \\
k
\end{array}\right) \widehat{G}_{2 k}(x):=\sum_{k=1}^{\infty} c(\alpha, k) \widehat{G}_{2 k}(x),
$$

where $G_{2 k}$ is the Bessel kernel of order $2 k$. Hence

$$
g_{\alpha}(x)=\sum_{k=1}^{\infty} c(\alpha, k) G_{2 k}(x), \quad x \in \mathbb{R}^{n} .
$$

Now, we stress that

$$
m_{\alpha}(x):=\sum_{k=1}^{\infty}|c(\alpha, k)| G_{2 k}(x)
$$

defines a radial decreasing dominant of $g_{\alpha}$. Furthermore, $m_{\alpha}$ is integrable,

$$
\left\|m_{\alpha}\right\|_{1} \leq \sum_{k=1}^{\infty}\left|\left(\begin{array}{c}
\alpha / 2 \\
k
\end{array}\right)\right|<\infty
$$

since $\left|\left(\begin{array}{c}\alpha / 2 \\ k\end{array}\right)\right| \leq \frac{c}{k^{1+\alpha / 2}}$ as $k \rightarrow \infty$ (cf. [26], p. 14).

As regards Lemma 5.4, we split its proof into two parts.

Step 1 (Proof of (5.6)): Let us start by representing the function $\widehat{h}_{\alpha}$ as a finite sum of Fourier transforms of Bessel kernels plus an integrable function. To this end, we denote $t=\frac{1}{1+|x|^{2}}$. Then $\widehat{h}_{\alpha}(x)=\frac{1}{t^{\beta}+(1-t)^{\beta}}-1$, with $\beta=\frac{\alpha}{2}$. But

$$
\begin{aligned}
\frac{1}{t^{\beta}+(1-t)^{\beta}}-1 & =\frac{1}{(1-t)^{\beta}} \cdot \frac{1}{1+\left(\frac{t}{1-t}\right)^{\beta}}-1 \\
& =\frac{1}{(1-t)^{\beta}} \sum_{k=0}^{\infty}(-1)^{k}\left(\frac{t}{1-t}\right)^{k \beta}-1
\end{aligned}
$$

where the series converges if $\frac{t}{1-t}<1$, that is, if $t<\frac{1}{2}$ or $|x|>1$. Since $\frac{1}{1-t}=\frac{1+|x|^{2}}{|x|^{2}}$ and $\frac{t}{1-t}=\frac{1}{|x|^{2}}$, we get

$$
\widehat{h}_{\alpha}(x)=\frac{\left(1+|x|^{2}\right)^{\frac{\alpha}{2}}}{|x|^{\alpha}} \sum_{k=0}^{\infty} \frac{(-1)^{k}}{|x|^{\alpha k}}-1, \quad|x|>1 .
$$


For each natural number $N$, we can write

$$
\widehat{h}_{\alpha}(x)=\left(1+|x|^{2}\right)^{\frac{\alpha}{2}} \sum_{k=0}^{N} \frac{(-1)^{k}}{|x|^{\alpha(k+1)}}-1+A_{N}(x), \quad|x|>1,
$$

where $\left|A_{N}(x)\right| \leq \frac{c}{|x|^{\alpha N}}$. Indeed, since $\frac{1}{|x|^{\alpha k}} \rightarrow 0$ as $k \rightarrow+\infty$ (recall that $|x|>1)$, we have

$$
\left|A_{N}(x)\right|=\left|\frac{\left(1+|x|^{2}\right)^{\frac{\alpha}{2}}}{|x|^{\alpha}} \sum_{k=N+1}^{\infty} \frac{(-1)^{k}}{|x|^{\alpha k}}\right| \leq \frac{\left(1+|x|^{2}\right)^{\frac{\alpha}{2}}}{|x|^{2 \alpha}} \frac{1}{|x|^{\alpha N}} \leq \frac{2^{\alpha}}{|x|^{\alpha N}} .
$$

Now it remains to represent the powers $\frac{1}{|x|^{\alpha(k+1)}}$ in terms of the powers $\frac{1}{\sqrt{1+|x|^{2}}}$. We observe that for any $\gamma>0$, taking $\rho=\sqrt{1+|x|^{2}}$, we have $(5.13)$

$$
\frac{1}{|x|^{\gamma}}=\rho^{-\gamma}\left(1-\frac{1}{\rho^{2}}\right)^{-\gamma / 2}=\rho^{-\gamma}\left(\sum_{j=0}^{M}(-1)^{j}\left(\begin{array}{c}
-\gamma / 2 \\
j
\end{array}\right) \rho^{-2 j}+\phi_{M}(\rho)\right),
$$

where $M \in \mathbb{N}$ and

$$
\phi_{M}(\rho)=\sum_{j=M+1}^{\infty}(-1)^{j}\left(\begin{array}{c}
-\gamma / 2 \\
j
\end{array}\right) \rho^{-2 j}
$$

converges absolutely for $\rho>1$, that is, for $x \neq 0$.

Obviously

$$
\left|\frac{\phi_{M}(\rho)}{\rho^{\gamma}}\right| \leq c \sum_{j=M+1}^{\infty} \frac{1}{j^{1-\frac{\gamma}{2}}} \frac{1}{\rho^{2 j+\gamma}} \leq \frac{c}{\rho^{M+1}} \sum_{j=M+1}^{\infty} \frac{1}{j^{1-\frac{\gamma}{2}}} \frac{1}{2^{\frac{j+\gamma}{2}}}
$$

where we took into account that $|x|>1 \Longleftrightarrow \rho \geq \sqrt{2}$. Hence

$$
\left|\frac{\phi_{M}(\rho)}{\rho^{\gamma}}\right| \leq \frac{c_{1}}{\rho^{M+1}} \leq \frac{c_{2}}{\rho^{M}} .
$$

Then from (5.13) and (5.15)

$$
\frac{1}{|x|^{\gamma}}=\sum_{j=0}^{M} \frac{(-1)^{j}\left(\begin{array}{c}
-\gamma / 2 \\
j
\end{array}\right)}{\left(1+|x|^{2}\right)^{j+\gamma / 2}}+B_{M}^{\gamma}(x),
$$


where

$$
\left|B_{M}^{\gamma}(x)\right| \leq \frac{C}{|x|^{2 M}} \quad \text { as } \quad|x|>1 .
$$

Substituting (5.16) into (5.11) (with $\gamma=\alpha(k+1)$ ), and taking $M=N$, we arrive at

$$
\widehat{h}_{\alpha}(x)=\sum_{\substack{k, j=0 \\
k+j \neq 0}}^{N} \frac{(-1)^{k+j}\left(\begin{array}{c}
-\alpha(k+1) / 2 \\
j
\end{array}\right)}{\left(1+|x|^{2}\right)^{j+\alpha k}}+r_{N}(x)
$$

where the function

$$
r_{N}(x)=A_{N}(x)+\left(1+|x|^{2}\right)^{\frac{\alpha}{2}} \sum_{k=0}^{N} B_{N}^{\alpha(k+1)}(x)
$$

satisfies the estimate

$$
\left|r_{N}(x)\right| \leq \frac{c}{|x|^{\mu}}, \quad \mu=N \min (2, \alpha),
$$

for all $|x|>1$ according to (5.12) and (5.17). Hence, we only have to choose $N>\frac{n}{\min (2, \alpha)}$ in order to get the integrability of $r_{N}$ at infinity.

The estimate at infinity was given for $|x|>1$, but the equality (5.18) itself may be written for all $x \in \mathbb{R}^{n}$, just by defining $r_{N}$ as

$$
r_{N}(x):=\widehat{h}_{\alpha}(x)-\sum_{\substack{k, j=0 \\ k+j \neq 0}}^{N} c(k, j) \widehat{G}_{2 j+\alpha k}(x), \quad N>\frac{n}{\min (2, \alpha)}, \quad x \in \mathbb{R}^{n},
$$

where $G_{2 j+\alpha k}$ are Bessel kernels and $c(k, j):=(-1)^{k+j}\left(\begin{array}{c}-\alpha(k+1) / 2 \\ j\end{array}\right)$.

So, we have $r_{N} \in \mathcal{W}_{0}$. In particular, $r_{N}$ is a bounded continuous function. Also, $r_{N}$ is integrable at infinity in view of the estimate above and hence, $r_{N} \in \mathcal{W}_{0} \cap L_{1}$. On the other hand, $F^{-1} r_{N} \in \mathcal{W}_{0} \cap L_{1}$. Thus, $F^{-1} r_{N}$ is a bounded continuous function too. So

$$
\begin{aligned}
\left|h_{\alpha}(x)\right| & \leq \sum_{\substack{k, j=0 \\
k+j \neq 0}}^{N}|c(k, j)|\left|G_{2 j+\alpha k}(x)\right|+\left|F^{-1} r_{N}(x)\right| \\
& \leq \sum_{\substack{k, j=0 \\
k+j \neq 0}}^{N}|c(k, j)|\left|G_{2 j+\alpha k}(x)\right|+C .
\end{aligned}
$$


We know that

$$
G_{2 j+\alpha k}(x) \sim \frac{1}{|x|^{n-2 j-\alpha k}} \leq \frac{c}{|x|^{n-\min (1, \alpha)}} \quad \text { as } \quad|x|<1,
$$

when $2 j+\alpha k<n$. Thus, we arrive at (5.6) with $a=\min (1, \alpha)$.

In the case $2 j+\alpha k>n$ we arrive at the same estimate since

$$
G_{2 j+\alpha k}(x) \sim C(2 j+\alpha k), \quad|x|<1 .
$$

For the case $2 j+\alpha k=n$ we have the following logarithmic behavior:

$$
G_{2 j+\alpha k}(x) \sim \ln \left(\frac{1}{|x|}\right), \quad|x|<1 .
$$

But $\ln \left(\frac{1}{|x|}\right) \leq \frac{1}{|x|^{n-a}}$ for any $a \in(0, n)$. The proof of (5.6) is completed.

Step 2 (Proof of (5.7)): To obtain (5.7), we transform the Bochner formula for the Fourier transform of radial functions via integration by parts and arrive at the formula

$$
F^{-1} \widehat{h}_{\alpha}(x)=\frac{c}{|x|^{\frac{n}{2}+m-1}} \int_{0}^{\infty} \psi_{\alpha}^{(m)}(t) t^{\frac{n}{2}} J_{\frac{n}{2}+m-1}(t|x|) d t, \quad x \neq 0
$$

where $\psi_{\alpha}(t)=\frac{\left(1+t^{2}\right)^{\frac{\alpha}{2}}}{1+t^{\alpha}}$ and $m$ is arbitrary such that $m>1+\frac{n}{2}$ (the latter condition on $m$ ) guarantees the convergence of the integral at infinity.

To justify formula (5.20), we make use of the standard regularization of the integral (cf. [30]):

$$
\begin{aligned}
F^{-1} \widehat{h}_{\alpha}(x) & =(2 \pi)^{-n} \lim _{\varepsilon \rightarrow 0} \int_{\mathbb{R}^{n}} e^{-\varepsilon|y|} e^{-i x \cdot y} \widehat{h}_{\alpha}(|y|) d y \\
& =(2 \pi)^{-n} \lim _{\varepsilon \rightarrow 0} \frac{(2 \pi)^{n / 2}}{|x|^{n / 2-1}} \int_{0}^{\infty} e^{-\varepsilon t} \widehat{h}_{\alpha}(t) t^{n / 2} J_{n / 2-1}(t|x|) d t \\
& =\frac{(2 \pi)^{-\nu}}{|x|^{\nu-1}} \lim _{\varepsilon \rightarrow 0} \int_{0}^{\infty} f_{\varepsilon}(t) t^{\nu} J_{\nu-1}(t|x|) d t \\
& =\frac{(2 \pi)^{-\nu}}{|x|^{\nu-1}} \lim _{\varepsilon \rightarrow 0} \frac{(-1)^{m}}{|x|^{m}} \int_{0}^{\infty} f_{\varepsilon}^{(m)}(t) t^{\nu} J_{\nu+m-1}(t|x|) d t
\end{aligned}
$$

where $J_{\nu-1}(t)$ denotes the Bessel function of the first kind, $\nu=\frac{n}{2}, m \in \mathbb{N}$ and

$$
f_{\varepsilon}(t):=e^{-\varepsilon t} \widehat{h}_{\alpha}(t), \quad \varepsilon>0 .
$$

The second equality follows from the Bochner formula for Fourier transforms of radial functions, while the last is obtained via integration by parts and the 
relation $\frac{d}{d u}\left[u^{\nu} J_{\nu}(u)\right]=u^{\nu} J_{\nu-1}(u)$ (cf. (8.133) in [24]). Here we assumed that some quantities vanish, namely

$$
\left.f_{\varepsilon}^{(k)}(t) t^{\nu} J_{\nu+k}(t|x|)\right|_{0} ^{\infty}=0, \quad k=0,1, \ldots, m-1 .
$$

To check this for $\psi_{\alpha}(t)=\varphi_{\alpha}(t) \cdot \phi_{\alpha}(t)$ with $\varphi_{\alpha}(t)=\left(1+t^{2}\right)^{\frac{\alpha}{2}}$ and $\phi_{\alpha}(t)=\frac{1}{1+t^{\alpha}}$, we observe that

$$
\varphi_{\alpha}^{(k)}(t)=\varphi_{\alpha}(t) \sum_{j=0}^{[k / 2]} c_{j}(\alpha) \frac{t^{k-2 j}}{\left(1+t^{2}\right)^{k-j}}, \quad k=0,1, \ldots
$$

and

$$
\phi_{\alpha}^{(k)}(t)=\phi_{\alpha}(t) t^{-k} \sum_{j=1}^{k} d_{j}(\alpha) \frac{t^{j \alpha}}{\left(1+t^{\alpha}\right)^{j}}, \quad k=1,2, \ldots
$$

where the constants $c_{j}(\alpha)$ and $d_{j}(\alpha)$ may vanish (but not all simultaneously), which may be directly proved. For $k \geq 1$, we have

$$
\begin{gathered}
\psi_{\alpha}^{(k)}(t)=\sum_{r=0}^{k}\left(\begin{array}{l}
k \\
r
\end{array}\right) \varphi_{\alpha}^{(r)}(t) \phi_{\alpha}^{(k-r)}(t) \\
=\psi_{\alpha}(t) \sum_{r=0}^{k-1}\left(\begin{array}{l}
k \\
r
\end{array}\right)\left(\sum_{j=0}^{[r / 2]} \frac{c_{j}(\alpha) t^{r-2 j}}{\left(1+t^{2}\right)^{r-j}}\right)\left(t^{-(k-r)} \sum_{j=1}^{k-r} \frac{d_{j}(\alpha) t^{j \alpha}}{\left(1+t^{\alpha}\right)^{j}}\right) \\
+\psi_{\alpha}(t)\left(\sum_{j=0}^{[k / 2]} \frac{c_{j}(\alpha) t^{k-2 j}}{\left(1+t^{2}\right)^{k-j}}\right) .
\end{gathered}
$$

Since $f_{\varepsilon}(t)=e^{-\varepsilon t}\left(\psi_{\alpha}(t)-1\right)$, then

$$
f_{\varepsilon}^{(k)}(t)=\sum_{j=0}^{k}\left(\begin{array}{l}
k \\
j
\end{array}\right)(-\varepsilon)^{k-j} e^{-\varepsilon t} \psi_{\alpha}^{(j)}(t)-(-\varepsilon)^{k} e^{-\varepsilon t} .
$$

Let $k \in\{0,1,2, \ldots, m-1\}$. Taking into account that $J_{\nu+k}(u)$ behaves like $u^{\nu+k}$ for small values of $u$, we obtain

$$
f_{\varepsilon}^{(k)}(t) t^{\nu} J_{\nu+k}(t|x|) \longrightarrow 0 \quad \text { as } \quad t \rightarrow 0 .
$$

On the other hand, $J_{\nu+k}(u)$ behaves like $\frac{1}{\sqrt{u}}$ for large values of $u$. Since $e^{-\varepsilon t} t^{\nu-1 / 2}$ goes to zero as $t \rightarrow \infty$ and $\left|\psi_{\alpha}^{(j)}(t)\right|$ behaves like a constant $(0$ 
or 1 , if $j>0$ or $j=0$, respectively) when $t \rightarrow \infty$, then

$$
f_{\varepsilon}^{(k)}(t) t^{\nu} J_{\nu+k}(t|x|) \longrightarrow 0 \quad \text { as } \quad t \rightarrow \infty,
$$

which completes the verification of (5.22).

To derive (5.20) from (5.21), we notice that the functions $f_{\varepsilon}^{(m)}(t) t^{\nu}$ $\times J_{\nu+m-1}(t|x|), \varepsilon>0$, are integrable in $(0, \infty)$. In fact, the integrability at the origin follows from the asymptotic behavior of the Bessel function, while its integrability at infinity follows from the definition of the Gamma function.

It suffices to note that $f_{\varepsilon}^{(m)}(t) \longrightarrow \psi_{\alpha}^{(m)}(t)$ as $\varepsilon \rightarrow 0$, by (5.23), and the passage to the limit in (5.21) is easily justified, which yields (5.20).

To obtain (5.7) from (5.20), we observe that the following estimates hold:

$$
\left|\psi_{\alpha}^{(m)}(t)\right| \leq \frac{c}{t^{m}} \quad \text { as } \quad t \geq 1
$$

and

$$
\left|\psi_{\alpha}^{(m)}(t)\right| \leq c\left(t^{\alpha-m}+t^{m-2\left[\frac{m}{2}\right]}\right) \quad \text { as } \quad t<1 .
$$

So, we have

$$
\begin{aligned}
& \frac{c}{|x|^{\nu+m-1}} \int_{0}^{1}\left|\psi_{\alpha}^{(m)}(t)\right| t^{\nu}\left|J_{\nu+m-1}(t|x|)\right| d t \\
& \leq \frac{c}{|x|^{\nu+m-1}} \int_{0}^{1} t^{\alpha-m+\nu}\left|J_{\nu+m-1}(t|x|)\right| d t \\
& \leq \frac{c}{|x|^{n+\alpha}} \int_{0}^{|x|} t^{\alpha-m+\nu}\left|J_{\nu+m-1}(t)\right| d t \\
& \leq \frac{c}{|x|^{n+\alpha}} \int_{0}^{\infty} t^{\alpha-m+\nu}\left|J_{\nu+m-1}(t)\right| d t=\frac{c_{1}}{|x|^{n+\alpha}}
\end{aligned}
$$

if $m>1+\nu+\alpha$, which guarantees the convergence of the last integral at infinity. The proof is completed.

\section{Connection of the Riesz and Bessel potentials with the Sobolev spaces of variable exponent}

The identification of the spaces of Bessel potentials of integer smoothness with Sobolev spaces is a well-known result within the framework of the classical Lebesgue spaces. The result is due to A. Calderón and states that $\mathcal{B}^{m}\left[L_{p}\right]=W_{p}^{m}$, if $m \in \mathbb{N}_{0}$ and $1<p<\infty$, with equivalent norms. We extend this to the variable exponent setting. The proof will follow mainly 
the case of constant $p$, which can be found, for instance, in [29], Sections V.3.3-4. In particular, we will make use of the Riesz transforms

$$
R_{j} f(x)=\lim _{\varepsilon \rightarrow 0} c_{n} \int_{|y|>\varepsilon} \frac{y_{j}}{|y|^{n+1}} f(x-y) d y, \quad j=1,2, \ldots, n .
$$

The key point is the following characterization:

Theorem 6.1. Let $p(\cdot) \in \mathcal{P}\left(\mathbb{R}^{n}\right)$ and let $\alpha \geq 1$. Then $f \in \mathcal{B}^{\alpha}\left[L_{p(\cdot)}\right]$, if and only if $f \in \mathcal{B}^{\alpha-1}\left[L_{p(\cdot)}\right]$ and $\frac{\partial f}{\partial x_{j}} \in \mathcal{B}^{\alpha-1}\left[L_{p(\cdot)}\right]$ for every $j=1, \ldots, n$. Furthermore, there exist positive constants $c_{1}$ and $c_{2}$ such that

$$
\begin{aligned}
c_{1}\left\|f \mid \mathcal{B}^{\alpha}\left[L_{p(\cdot)}\right]\right\| & \leq\left\|f\left|\mathcal{B}^{\alpha-1}\left[L_{p(\cdot)}\right]\left\|+\sum_{j=1}^{n}\right\| \frac{\partial f}{\partial x_{j}}\right| \mathcal{B}^{\alpha-1}\left[L_{p(\cdot)}\right]\right\| \\
& \leq c_{2}\left\|f \mid \mathcal{B}^{\alpha}\left[L_{p(\cdot)}\right]\right\| .
\end{aligned}
$$

Proof. Suppose first that $f=\mathcal{B}^{\alpha} \varphi$ for some $\varphi \in L_{p(\cdot)}$. Then for each $j=1,2, \ldots, n$, we have

$$
\frac{\partial f}{\partial x_{j}}=\mathcal{B}^{\alpha-1}\left[-R_{j}\left(I+K_{1}\right) \varphi\right]
$$

where $I$ is the identity operator and $K_{1}$ is the convolution operator whose kernel is $g_{1}$, given by (5.3) with $\alpha=1$. This identity, obvious in Fourier transforms, is known to be valid for $\varphi \in L_{p}$ when $p$ is constant, see [29], p. 136. Then it is also valid for variable $p(\cdot)$, since $L_{p(\cdot)} \subset L_{\bar{p}}+L_{\underline{p}}$.

The right-hand side inequality in (6.1) follows from (6.2) and from the mapping properties of the Bessel potential operator on spaces $L_{p(\cdot)}$.

The proof of the left-hand side inequality follows the known scheme for constant $p$. However, we need to refine the connection with the Riesz transforms and the derivatives, in order to overcome the difficulties associated to the convolution operators in the variable exponent setting. We write here the main steps of the proof for the completeness of the presentation.

Assume that both $f$ and $\frac{\partial f}{\partial x_{j}}$ belong to $\mathcal{B}^{\alpha-1}\left[L_{p(\cdot)}\right]$. If $f=\mathcal{B}^{\alpha-1} \varphi$, with $\varphi \in L_{p(\cdot)}$, then the first order derivatives of $\varphi$ exist in the weak sense and belong to $L_{p(\cdot)}$. Moreover, $\frac{\partial f}{\partial x_{j}}=\mathcal{B}^{\alpha-1}\left(\frac{\partial \varphi}{\partial x j}\right)$. Since $\varphi \in W_{p(\cdot)}^{1}$ there exists a sequence of infinitely differentiable and compactly supported functions $\left\{\varphi_{k}\right\}_{k \in \mathbb{N}}$ such that $\lim _{k \rightarrow \infty} \varphi_{k}=\varphi$ and $\lim _{k \rightarrow \infty} \frac{\partial \varphi_{k}}{\partial x_{j}}=\frac{\partial \varphi}{\partial x_{j}}$ in $L_{p(\cdot)}$, $j=1,2, \ldots, n$. This follows from the denseness of $C_{0}^{\infty}$ in the Sobolev space $W_{p(\cdot)}^{1}$ (see [23]), which holds under the assumptions on the exponent. This completes the proof. 
Corollary 6.2. Let $p(\cdot)$ be as in Theorem 6.1 and let $m \in \mathbb{N}_{0}$. Then

$$
\mathcal{B}^{m}\left[L_{p(\cdot)}\right]=W_{p(\cdot)}^{m},
$$

up to the equivalence of the norms.

The theorem below provides a connection of the spaces of Riesz potentials with the Sobolev spaces. It partially extends the facts known for constant $p$ (see, for instance, [24], p. 181) to the variable exponent setting.

Theorem 6.3. Let $p(\cdot)$ be $\log$-Hölder continuous both locally and at infinity, with $1<\underline{p} \leq \bar{p}<n / \alpha$. Then we have

$$
W_{p(\cdot)}^{m} \subset L_{p(\cdot)} \cap \mathcal{I}^{\alpha}\left[L_{p(\cdot)}\right]
$$

if $0<\alpha<\min (m, n), m \in \mathbb{N}$, and

$$
W_{p(\cdot)}^{m}=L_{p(\cdot)} \cap \mathcal{I}^{m}\left[L_{p(\cdot)}\right]
$$

when $0<m<n$.

Proof. Let us prove (6.4) first. Let $f \in W_{p(\cdot)}^{m}$. From Corollary 6.2, Proposition 5.2 and Theorem 5.7, we derive that not only $f \in L_{p(\cdot)}$, but also that $\mathbb{D}^{m} f \in L_{p(\cdot)}$. On the other hand, the Sobolev theorem states that $f \in L_{q(\cdot)}$, where $q(\cdot)$ is the usual Sobolev exponent. Then by Theorem 3.2 one concludes that $f$ is a Riesz potential. Reciprocally, if $f \in \mathcal{I}^{m}\left[L_{p(\cdot)}\right]$ then the application of Theorem 3.2 shows that $\mathbb{D}^{m} f$ exists in $L_{p(\cdot)}$, which implies $f \in L_{p(\cdot)}^{m}$. As above, one gets $f \in W_{p(\cdot)}^{m}$.

The embedding (6.3) can be proved following similar arguments by observing that $\mathcal{B}^{m}\left[L_{p(\cdot)}\right] \hookrightarrow \mathcal{B}^{\alpha}\left[L_{p(\cdot)}\right]$ when $m>\alpha$.

\section{References}

[1] A. Almeida, Inversion of the Riesz potential operator on Lebesgue spaces with variable exponent, Fract. Calc. Appl. Anal., 6 (3) (2003), 311-327.

[2] C. Capone, D. Cruz-Uribe and A. Fiorenza, The fractional maximal function on variable $L^{p}$ spaces, Preprint, (2004); available at www.na.iac.cnr.it.

[3] D. Cruz-Uribe, A. Fiorenza and C. J. Neugebauer, The maximal function on variable $L^{p}$ spaces, Ann. Acad. Sci. Fenn. Math., 28 (2003), 223-238.

[4] D. Cruz-Uribe, A. Fiorenza, J. M. Martell and C. Pérez, The boundedness of classical operators on variable $L^{p}$ spaces, Preprint (2004); available at www.na.iac.cnr.it. 
[5] L. Diening, Maximal function on generalized Lebesgue spaces $L^{p(\cdot)}$, Math. Inequal. Appl., 7 (2) (2004), 245-253.

[6] L. Diening, Maximal function on Musietak-Orliz spaces and generalized Lebesgue spaces, Preprint, 2004.

[7] L. Diening, Riesz potential and Sobolev embeddings on generalized Lebesgue and Sobolev spaces $L^{p(\cdot)}$ and $W^{k, p(\cdot)}$, Math. Nachr., 268 (2004), 31-43.

[8] L. Diening and M. RůžIčka, Calderón-Zygmund operators on generalized Lebesgue spaces $L^{p(\cdot)}$ and problems related to fluid dynamics, J. Reine Angew. Math., 563 (2003), 197-220.

[9] D. E. Edmunds and J. Rákosník, Density of smooth functions in $W^{k, p(x)}(\Omega)$, Proc. Roy. Soc. London Ser. A, 437 (1992), 229-236.

[10] X. Fan, J. Shen and D. Zhao, Sobolev embedding theorems for spaces $W^{k, p(x)}(\Omega)$, J. Math. Anal. Appl., 262 (2) (2001), 749-760.

[11] P. Harjulehto and P. Hästö, Lebesgue points in variable exponent spaces, Ann. Acad. Sci. Fenn. Math., 29 (2) (2004), 295-306.

[12] V. Kokilashvili, On a Progress in the Theory of Integral Operators in Weighted Banach Function Spaces, (to appear in Proceed. of Conference Funct. Spaces, Diff. Oper., Nonlin. Anal. - FSDONA 2004 dedicated to the 70th birthday of Prof. Alois Kufner, May 27 - June 2, 2004 Svratka, Czech Republic).

[13] V. Kokilashvili and S. Samko, Maximal and fractional operators in weighted $L^{p(x)}$ spaces, Rev. Mat. Iberoamericana, 20 (2) (2004), 493515.

[14] V. Kokilashvili and S. Samko, On Sobolev theorem for Riesz type potentials in Lebesgue spaces with variable exponent, Z. Anal. Anwend., 22 (4) (2003), 899-910.

[15] V. Kokilashvili and S. Samko, Singular integrals in weighted Lebesgue spaces with variable exponent, Georgian Math. J., 10 (1) (2003), 145156.

[16] V. Kovácik and J. Rákosník, On spaces $L^{p(x)}$ and $W^{k, p(x)}$, Czech. Math. J., 41 (116) (1991), 592-618.

[17] P. I. Lizorkin, Generalized Liouville differentiation and the functional spaces $L_{p}^{r}\left(E_{n}\right)$. Imbedding theorems (Russian), Math. Sb., 60 (1963), $325-353$.

[18] A. Nekvinda, Hardy-Littlewood maximal operator on $L^{p(x)}\left(\mathbb{R}^{n}\right)$, Math. Inequal. Appl., 7 (2) (2004), 255-265.

[19] M. Růžička, Electrorheological fluids: modeling and mathematical theory, Lecture Notes in Mathematics, 1748, Springer, Berlin 2000.

[20] S. G. Samko, Spaces of Riesz potentials (in Russian), Izv. Akad. Nauk SSSR, Ser. Mat., 40 (5) (1976), 1143-1172.

[21] S. G. Samko, Convolution type operators in $L^{p(x)}\left(\mathbb{R}^{n}\right)$, Integral Transform. Spec. Funct., 7 (1-2) (1998), 123-144. 
[22] S. G. Samko, Convolution and potential type operators in $L^{p(x)}\left(\mathbb{R}^{n}\right)$, Integral Transform. Spec. Funct., 7 (3-4) (1998), 261-284.

[23] S. G. Samko, Denseness of $C_{0}^{\infty}\left(\mathbb{R}^{N}\right)$ in the generalized Sobolev spaces $W^{M, P(X)}\left(\mathbb{R}^{N}\right)$, In : Direct and Inverse Problems of Mathematics Physics (Newark, DE, 1997), Int. Soc. Anal. Appl. Comput., 5, 333342, Kluwer Acad. Publ. Dordrecht, 2000.

[24] S. G. Samko, Hypersingular integrals and their applications, Ser. Analytical Methods and Special Functions, Taylor \& Francis, London 2002.

[25] S. G. Samko, On a progress in the theory of Lebesgue spaces with variable exponent: maximal and singular operators, Integral Transforms Spec. Funct. 16 (5-6) (2005), 461-482.

[26] S. G. Samko, A. A. Kilbas and O. I. Marichev, Fractional Integrals and Derivatives: Theory and Applications, Gordon and Breach, New York 1993.

[27] I. I. Sharapudinov, On a topology of the space $L^{p(t)}([0,1])$, Math. Notes, 26 (1979), 796-806.

[28] E. Stein, The characterization of functions arising as potentials, I, Bull. Amer. Math. Soc., 67 (1) (1961), 102-104.

[29] E. Stein, Singular Integrals and Differentiability Properties of Functions, Princeton University Press, Princeton 1970.

[30] E. Stein and G. Weiss, Introduction to Fourier Analysis on Euclidean Spaces, Princeton University Press, Princeton 1971.

Departamento de Matemática

Universidade de Aveiro

3810-193 Aveiro

Portugal

(E-mail : alexandre@mat.ua.pt)

Faculdade de Ciências e Tecnologia

Universidade do Algarve

Campus de Gambelas

8005-139 Faro

Portugal

(E-mail : ssamko@ualg.pt)

(Received : March 2005) 


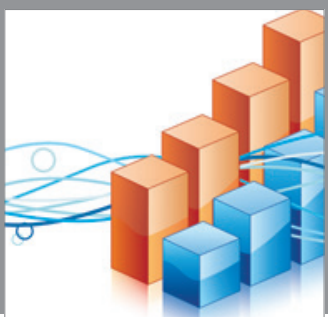

Advances in

Operations Research

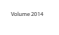

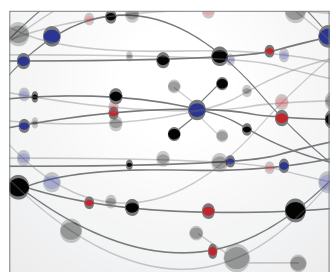

\section{The Scientific} World Journal
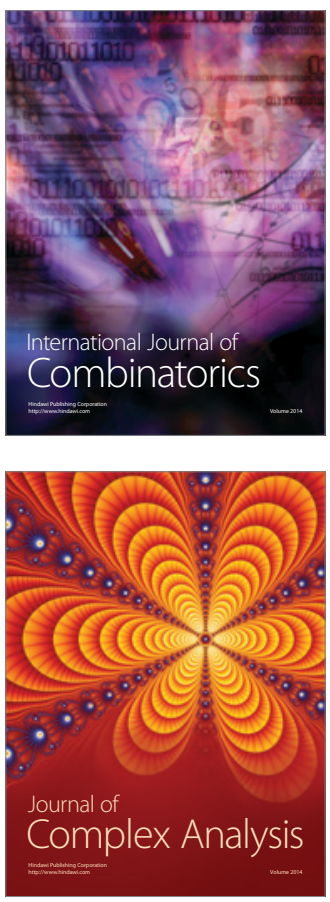

International Journal of

Mathematics and

Mathematical

Sciences
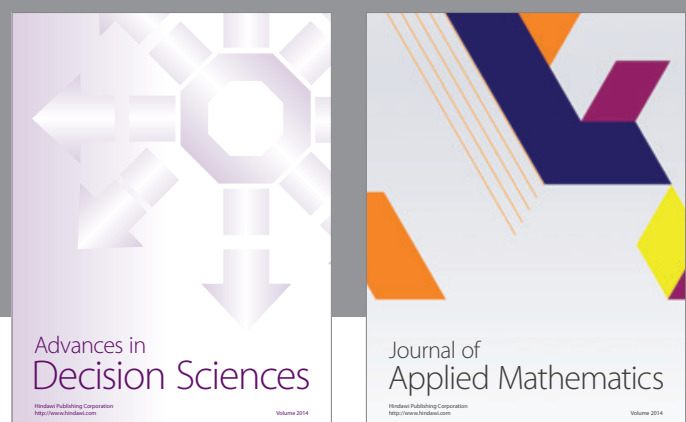

Journal of

Applied Mathematics
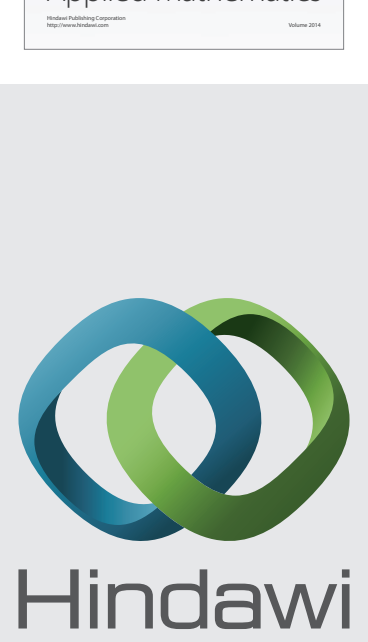

Submit your manuscripts at http://www.hindawi.com
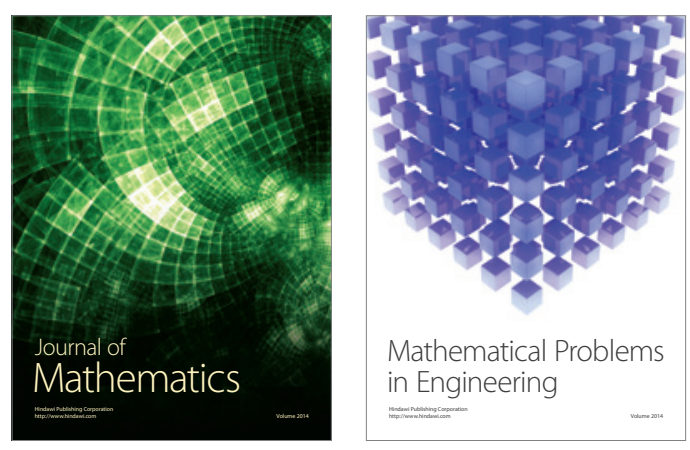

Mathematical Problems in Engineering
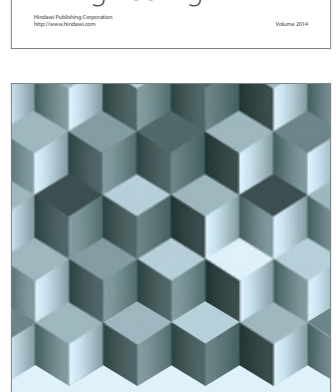

Journal of

Function Spaces
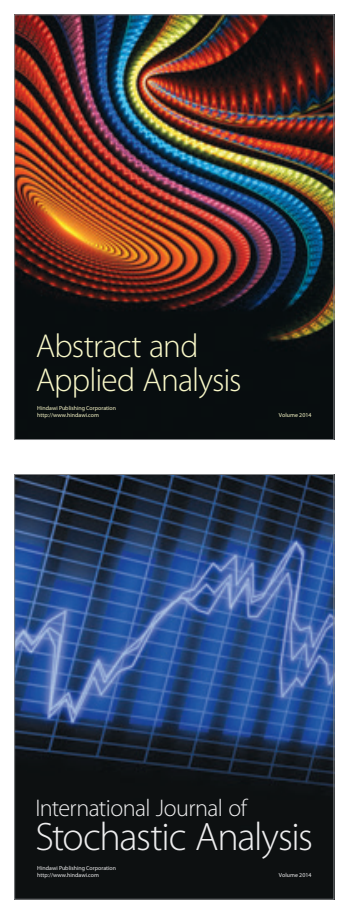

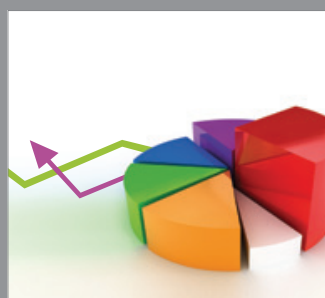

ournal of

Probability and Statistics

Promensencen
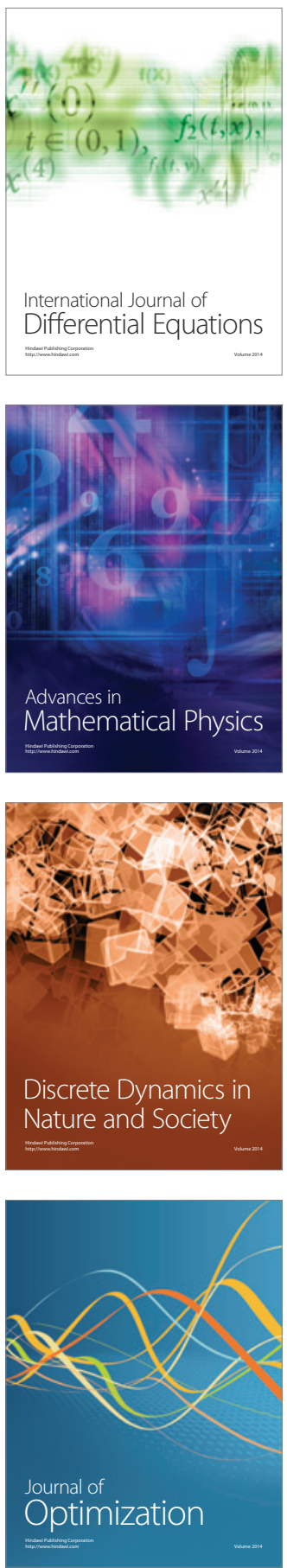